\title{
A two-phase solver for complex fluids: Studies of the Weissenberg effect
}

\author{
R.A. Figueiredo ${ }^{\text {, }}$, C.M. Oishi ${ }^{\text {b,* }}$, A.M. Afonso ${ }^{c}$, I.V.M. Tasso ${ }^{a}$, J.A. Cuminato ${ }^{a}$ \\ a Instituto de Ciências Matemáticas e Computação (ICMC), Universidade de São Paulo Av. do Trabalhador São-Carlense, 400, São Carlos - SP, Brazil \\ ${ }^{\mathrm{b}}$ Departamento de Matemática e Computação, Faculdade de Ciências e Tecnologia Universidade Estadual Paulista Júlio de Mesquita Filho, Presidente \\ Prudente 19060-900, SP, Brazil \\ ${ }^{c}$ CEFT- Centro de Estudos de Fenómenos de Transporte, Faculdade de Engenharia da Universidade do Porto, Rua Dr. Roberto Frias s/n, Porto, 4200-465, \\ Portugal
}

\section{A R T I C L E I N F O}

\section{Article history:}

Received 30 October 2015

Revised 12 April 2016

Accepted 17 April 2016

Available online 22 April 2016

\section{Keywords:}

Two-phase solver

Viscoelastic flow

Finite difference method

Volume-of-Fluid

Weissenberg effect

\begin{abstract}
A B S T R A C T
In this work a new two-phase solver is presented and described, with a particular interest in the solution of highly elastic flows of viscoelastic fluids. The proposed code is based on a combination of classical Volume-of-Fluid and Continuum Surface Force methods, along with a generic kernel-conformation tensor transformation to represent the rheological characteristics of the (multi)-fluid phases. Benchmark test problems are solved in order to assess the numerical accuracy of distinct levels of physical complexities, such as the interface representation, the influence of advection schemes, the influence of surface tension and the role of fluid rheology. In order to demonstrate the new features and capabilities of the solver in simulating of complex fluids in transient regime, we have performed a set of simulations for the problem of a rotating rod inserted into a container with a viscoelastic fluid, known as the Weissenberg or RodClimbing effect. Firstly, our results are compared with numerical and experimental data from the literature for low angular velocities. Secondly, we have presented results obtained for high angular velocities (high elasticity) using the Oldroyd-B model which displayed very elevated climbing heights. Furthermore, above a critical value for the angular velocity, it was observed the onset of elastic instabilities driven by the combination of elastic stresses, interfacial curvature and secondary flows, that to the authors best knowledge, were not yet reported in the literature.
\end{abstract}

(c) 2016 Elsevier Ltd. All rights reserved.

\section{Introduction}

Direct numerical simulations of multiphase flows, in which the full continuum equations are solved on a sufficiently refined computational mesh to resolve all continuum scales, date back to the origin of computational fluid dynamics. During the last decades, however, major progresses have been made, by employing a variety of numerical techniques for modeling two-phase flows, either using Lagrangian, Eulerian or Arbitrary Lagrangian-Eulerian (ALE) methodologies. Lagrangian and ALE methods usually represent interfaces accurately, but are rather complex to implement using mesh-dependent discretizations, due to the large mesh distortions involved in fluid flows (Quan, 2011; Montefuscolo et al., 2014). In the ALE method, the mesh follows the interface between the fluid and the solid boundary and the governing equations are discretized

\footnotetext{
* Corresponding author.

E-mail addresses: raf@icmc.usp.br (R.A. Figueiredo), oishi@fct.unesp.br, cassiooishi@gmail.com (C.M. Oishi), aafonso@fe.up.pt (A.M. Afonso), italo@tasso.com.br (I.V.M. Tasso), jacumina@icmc.usp.br (J.A. Cuminato).
}

on a moving mesh. ALE algorithms require a mesh deformation strategy as the boundaries of the computational domain translate, rotate and deform in order to maintain mesh quality and validity. On the other hand, Eulerian methods are faster and easily parallelizable, but suffer from inherent difficulties for the accuracy of the interface representation, that has to be immersed on the fixed grid. One of the issues of these Eulerian techniques is the increased importance of surface effects (surface tension, Marangoni effects, etc.), quantified by very low capillary $(\mathrm{Ca})$ or Weber $(\mathrm{We})$ numbers. In such cases, the inaccuracies in representing the exact position of the immersed interface generate approximations that are unbalanced, usually between surface, inertial and viscous forces, resulting in the so-called "parasitic currents", a phenomenon that is well documented in the literature Raessi et al. (2009). Additionally, time-step restrictions are a major concern. Since most of interface representations in Eulerian formulations are explicit in time, these restrictions prevent the simulation of real material properties, to the point that recent multiphase codes cannot simulate flows with Reynolds number $(R e)$ and $C a$ much lower than $O\left(10^{-2}\right)$ (Hoang et al., 2013; Denner and van Wachem, 2015). Existing 
Eulerian approaches to multiphase flows involve either marker particles, like in Front-tracking techniques (Tryggvason et al., 2001; Pivello et al., 2014), or marker functions as in the Level-set (LS) (Sussman et al., 1994; Scardovelli and Zaleski, 1999; Osher and Fedkiw, 2001; Sussman et al., 1998; Van der Pijl et al., 2005) and Volume-of-Fluid (VOF) (Hirt and Nichols, 1981; Pilliod and Puckett, 2004) approaches. In order to obtain improved interface position and geometric properties such as curvature and normal vector (needed to compute surface tension), hybrid techniques are also available in the literature (Sussman and Puckett, 2000; Sussman et al., 2007), benefiting from the accurate mass conservation of the VOF method and the smooth interface description provided by the LS method.

In recent years, Eulerian mesh-based methodologies have been used for solving interfacial complex fluid flows. Complexity does not arise only from the interaction of different fluid, but also from the physics that governs important phenomena. In some cases, more complex behaviors arise via rheological effects introduced by non-Newtonian and viscoelastic fluids. Many attempts for solving two-phase viscoelastic fluid flows have been presented in the literature see for instance (Pillapakkam and Singh, 2001; Yue et al., 2005; Khismatullin et al., 2006; Stewart et al., 2008; Lind and Phillips, 2010; Habla et al., 2011; Izbassarov and Muradoglu, 2015). Despite of this increase in the development of numerical methods to deal with two-phase viscoelastic fluid flows, there is still unsolved numerical issues and challenges. In non-Newtonian fluids, elastic instabilities can occur even in the absence of inertia, associated with large normal stresses and curvature of streamlines. From a computational perspective, these instabilities present a demanding challenge, such as the High Weissenberg Number Problem (HWNP), leading to the loss of convergence at very low level of elasticity, quantified by the Weissenberg number (Wi). Such numerical failure is usually attained at moderately low Weissenberg numbers $(W i \sim 1)$. This is particularly significant for multi-phase flows, where representing and tracking an interface with complex shape and dynamics are quite challenging. Therefore, the combination of classical numerical methods to represent the interface, surface tension and curvature, along with stabilization techniques to handle the HWNP, can be considered as an useful and innovative framework in current computational rheology.

The main objectives of this work are two-folded: (i) present to the scientific community, a validated new two-phase solver that can deal with multi-phase flows and fluids of complex rheology, and (ii) report interesting results obtained for the Weissenberg effect, related to the dynamical aspects of the onset of elastic instabilities and unsteady flow patterns formed at high rod angular velocities. As far as we are aware, such flow features have not been reported in previous studies based on numerical simulations and it is the ability of this new solver that makes these predictions possible. This numerical framework combines the classic VOF and Continuum surface force (VOF-CSF) and the Height Function (HF) method, along with the generic kernel-conformation tensor transformations. To the authors knowledge, in the context of viscoelastic two-phase flows, it is the first work that describes the implementation of the VOF method, using a piecewise linear interface construction method (PLIC) to reconstruct the interface and a least squares VOF interface reconstruction algorithm (ELVIRA), for solving the HWNP. The code is verified with several benchmark tests, as the viscoelastic laminar lid-driven cavity flow, the axisymmetric concentric annulus with inner cylinder rotation and the droplet spreading of a viscoelastic fluid. Finally, we performed a set of simulations for the application problem of a rotating rod inserted into a container with viscoelastic fluid, named the Weissenberg or RodClimbing effect.

The paper is organized as follows. The governing equations used to define the dynamics of an isothermal and incompressible flow of complex multi-fluids are discussed in Section 2. Section 3 describes the numerical algorithms used in the finite difference code. The validation of the numerical formulations are presented in Section 4. The obtained results and corresponding discussions of the numerical simulations for the Weissenberg effect are presented in Section 5. Finally, the conclusions from this study are presented in Section 6.

\section{Governing equations}

The flow is assumed to be isothermal, laminar, and the fluids incompressible. The governing equations are those expressing conservation of mass

$\nabla \cdot \mathbf{u}=0$

and conservation of momentum

$\rho\left(\frac{\partial \mathbf{u}}{\partial t}+\mathbf{u} \cdot \nabla \mathbf{u}\right)=\nabla \cdot\left(-p \mathbf{I}+\boldsymbol{\tau}+2 \mu_{s} \mathbf{D}\right)+\mathbf{g}+\mathbf{F}$,

where $\mathbf{u}$ is the velocity field, $t$ is time, $\rho$ is the density, $\mu_{s}$ is the Newtonian solvent viscosity, $p$ is the pressure, $\mathbf{g}$ is the gravity force and $\mathbf{F}$ is the surface tension force. The symbol $\mathbf{D}=\frac{1}{2}\left(\nabla \mathbf{u}+(\nabla \mathbf{u})^{T}\right)$ is the rate of deformation tensor, $\boldsymbol{\tau}$ is the elastic stress and $\mathbf{I}$ is the identity tensor.

Several polymeric constitutive equations are implemented in the current version of the solver: the Oldroyd-B model (Bird et al., 1987), the linear form of the Phan-Thien-Tanner (LPTT) model (Phan-Thien and Tanner, 1977) and the Giesekus model (Giesekus, 1982). For an isothermal flow, these rheological equations of state can be written in a compact form as:

$\frac{\partial \boldsymbol{\tau}}{\partial t}+(\mathbf{u} \cdot \nabla) \boldsymbol{\tau}-\left[(\nabla \mathbf{u})^{T} \cdot \boldsymbol{\tau}+\boldsymbol{\tau} \cdot \nabla \mathbf{u}\right]=\frac{1}{\lambda} \mathbf{M}(\boldsymbol{\tau})$,

where $\mathbf{M}(\boldsymbol{\tau})$ is defined by the viscoelastic model

$\mathbf{M}(\boldsymbol{\tau})=\left\{\begin{array}{lr}2 \mu_{p} \mathbf{D}-\boldsymbol{\tau} & \text { Oldroyd-B, } \\ 2 \mu_{p} \mathbf{D}-\boldsymbol{\tau}-\frac{\alpha \lambda}{\mu_{p}} \boldsymbol{\tau} \cdot \boldsymbol{\tau} & \text { Giesekus, } \\ 2 \mu_{p} \mathbf{D}-\left(1+\frac{\epsilon \lambda}{\mu_{p}} \operatorname{tr}(\boldsymbol{\tau})\right) \boldsymbol{\tau} & \text { LPTT, } \\ -\lambda \xi(\boldsymbol{\tau} \cdot \mathbf{D}+\mathbf{D} \cdot \boldsymbol{\tau}) & \end{array}\right.$

where $\lambda$ is a relaxation time and $\mu_{p}$ is the polymer viscosity coefficient. The stress coefficient function of the LPTT model, depends on the trace of $\boldsymbol{\tau}, \operatorname{tr}(\boldsymbol{\tau})$, and introduces the dimensionless parameter $\epsilon$ which is closely related to the steady-state elongational viscosity in extensional flows. The slip parameter, $\xi$, takes into account the non-affine motion between the polymer molecules and the continuum. The polymer strands embedded in the medium may slip with respect to the deformation of the macroscopic medium, thus each strand may transmit only a fraction of its tension to the surrounding continuum. When $\xi=0$ there is no slip and the motion becomes affine. The parameter $\xi$ is responsible for a non-zero second normal-stress difference in shear, leading to secondary flows in ducts having non-circular cross-sections, which is superimposed on the streamwise flow. In the nonlinear term of the Giesekus model, $\alpha$ represents a dimensionless "mobility factor". The amount of Newtonian solvent is controlled by the dimensionless solvent viscosity coefficient, $\beta=\frac{\mu_{S}}{\mu_{0}}$, where $\mu_{0}=\mu_{S}+\mu_{P}$ denotes the total shear viscosity.

An alternative form of describing viscoelastic models is by using the conformation tensor, A, as proposed by Fattal and Kupferman (2005). In this formulation the velocity gradient is defined as

$\nabla \mathbf{u}^{\mathbf{T}}=\mathbf{\Omega}+\mathbf{B}+\mathbf{N A}^{-1}$,

where $\boldsymbol{\Omega}$ and $\mathbf{N}$ are anti-symmetric tensors, $\mathbf{B}$ is symmetric and commutes with $\mathbf{A}$. Thus the constitutive equation based on the 
conformation tensor can be rewritten using Eq. (5) as

$\frac{\partial \mathbf{A}}{\partial t}+(\mathbf{u} \cdot \nabla) \mathbf{A}-(\boldsymbol{\Omega} \mathbf{A}-\mathbf{A} \boldsymbol{\Omega})-2 \mathbf{B} \mathbf{A}=\frac{1}{\lambda} \mathcal{M}(\mathbf{A})$,

where $\mathcal{M}(\mathbf{A})$ is defined according to the viscoelastic model,

$\mathcal{M}(\mathbf{A})=\left\{\begin{array}{lr}\mathbf{I}-\mathbf{A} & \begin{array}{r}\text { Oldroyd-B, } \\ \mathbf{I}-\mathbf{A}-\alpha(\mathbf{A}-\mathbf{I}) \cdot(\mathbf{A}-\mathbf{I})\end{array} \\ \left(1+\frac{\epsilon \lambda}{\mu_{p}}(\operatorname{tr}(\mathbf{A})-3)\right)(\mathbf{I}-\mathbf{A}) & \text { Giesekus, } \\ -2 \lambda \xi(\mathbf{B}-\mathbf{B A}) & \end{array}\right.$

The relationship between the stress tensor $\boldsymbol{\tau}$ and $\mathbf{A}$ is given by

$\boldsymbol{\tau}=\frac{\mu_{p}}{\lambda}(\mathbf{A}-\mathbf{I})$.

For non-Newtonian fluids, elastic instabilities can occur even in the absence of inertia, associated with large normal stresses and curvature of streamlines. When the elastic instabilities become very intense, reaching a saturated non linear state, fluctuations can even become random over a wide range of length and time scales, very much like turbulence, in spite of small Reynolds numbers. From a computational perspective, these instabilities present a demanding challenge, such as the High Weissenberg Number Problem, leading to the loss of convergence at very low level of elasticity, quantified by the Weissenberg number, $W i=\lambda / t_{c}$, where $t_{c}$ is a characteristic time of the flow. Such numerical breakdown is usually attained at moderately low Weissenberg numbers (Wi $\sim 1$ ). In order to implement a stabilization method to deal with high values of the Weissenberg number, we have adopted the generic kernel-conformation tensor transformation (Afonso et al., 2012) where several matrix kernel-transformation families can be applied to the conformation tensor evolution Eq. (6). The kernelconformation constitutive law is obtained by introducing the following kernel-conformation tensor transformation,

$\mathbb{k}(\mathbf{A})=\mathbf{O} \mathbb{k}(\boldsymbol{\Lambda}) \mathbf{0}^{T}$,

where $\mathbb{k}()$ represents a continuous, invertible and differentiable matrix function. Notice that this transformation is mathematically possible due to the fact that the conformation tensor can be diagonalized into:

$\mathbf{A}=\mathbf{0} \boldsymbol{\Lambda} \mathbf{0}^{T}$,

where $\mathbf{O}$ is an orthogonal matrix and $\boldsymbol{\Lambda}$ is a diagonal matrix.

In summary, the evolution equation used for the kernelconformation tensor transformation is given by:

$\frac{\mathrm{D} \mathbb{k}(\mathbf{A})}{\mathrm{D} t}=\boldsymbol{\Omega} \mathbb{k}(\mathbf{A})-\mathbb{k}(\mathbf{A}) \boldsymbol{\Omega}+2 \mathbb{B}+\frac{1}{\lambda} \mathbb{M}$

where $\mathbb{B}$ and $\mathbb{M}$ are symmetric tensors constructed by an orthogonalization process using Eqs. (5) and (7), respectively. More details about this formulation can be found in Afonso et al., 2012 and Martins et al., 2015.

In order to represent the interface between different fluids, the VOF method is used, in which, a function $f$ with values in $[0,1]$, known as volume fraction, is transported by the following expression:

$\frac{\partial f}{\partial t}+\nabla \cdot(f \mathbf{u})=0$.

The volume fraction is defined in each computational cell and the fluid properties are interpolated at the interface cells as

$\chi=f \chi_{1}+(1-f) \chi_{2}$,

where $\chi$ can be any property of the fluid such as $\rho, \mu_{s}, \mu_{p}, \lambda, \epsilon$, $\xi$ and $\alpha$, with the subscripts 1 and 2 representing the properties of each phase of the fluid.
The interface representation used in the present work for viscoelastic two-phase simulation is based on the VOF method, using the PLIC (Youngs, 1982) to reconstruct the interface together with the ELVIRA (Pilliod and Puckett, 2004). Moreover, the surface tension $\mathbf{F}$ term in the momemtum Eq. (2) is modeled by the CSF method (Brackbill et al., 1992). This method considers the surface tension term as a surface force per unit area of the interface as

$\mathbf{F}=\sigma \kappa \mathbf{n} \delta_{i}$,

where $\sigma$ is the surface tension coefficient, $\kappa$ is the interface curvature, $\mathbf{n}$ is the normal vector to the interface and $\delta_{i}$ is the $\delta$ function at the interface. In Eq. (14) some terms are computed using the volume fraction, for instance $\mathbf{n}=\nabla f /|\nabla f|$ and $\delta_{i}=|\nabla f|$, while the curvature is given by:

$\kappa=\kappa_{1}+\kappa_{2}=r_{1}^{-1}+r_{2}^{-1}$,

where $r_{1}$ and $r_{2}$ are the radius of curvature. In axisymmetric coordinates $\kappa_{1}$ is calculated in the $r-z$ plane by the volume fraction according to the HF method (Francois et al., 2006).

From the above equations, five dimensionless parameters are defined, that relate the properties of the fluid with the geometric parameters of the flow; namely, the Reynolds number $R e$, the Weissenberg number Wi, the Froude number Fr, the Stokes number $S t$ and the Capillary number $\mathrm{Ca}$, defined by

$R e=\frac{\rho U L}{\mu_{0}}, \quad W i=\lambda \frac{U}{L}, \quad F r=\frac{U}{\sqrt{g L}}, \quad S t=\frac{\mu_{0} U}{\rho g}, \quad C a=\frac{\mu_{0} U}{\sigma}$,

where $L$ and $U$ are appropriate length and velocity scales and $g$ is the magnitude of the gravity field.

\section{Numerical method}

The numerical solver proposed in this work is based on a finite difference method, in which the Navier-Stokes Eqs. (1) and (2) are discretized in non-uniform staggered grids. To obtain the velocity and pressure fields, a semi-implicity time discretization of the projection method is used. Further details on the numerical implementation for these equations are presented in Section 3.1. The extra-stress tensor in the momentum equations is obtained via an explicit time discretization of the constitutive equations, relying on both the standard extra-stress formulation - Eq. (3), the tensor conformation formulation - using Eq. (6) or other matrix transformations (11), as detailed in Section 3.2. Finally, details about the application of the non-uniform mesh are described in Section 3.3.

\subsection{Time discretization and the projection method}

Several viscoelastic flows effects usually occur in low Reynolds and high Weissenberg numbers regimes. This creates a problem for explicit methods because the time step is defined as a function of the Reynolds number due to the parabolic stability restriction. To circumvent this restriction we have used an implicit time discretization of the diffusive terms in Eq. (2) while the convective terms are explicitly computed. In particular, the numerical approach used in the present work, for solving the NavierStokes equations Eqs. (1) and (2) is an adaptation of the projection method described in Figueiredo et al., 2013 for the solution of viscoelastic fluid flows. Summarily, we have:

$\nabla \cdot \mathbf{u}^{n+1}=0$,

$$
\begin{aligned}
& \rho\left(\frac{\mathbf{u}^{n+1}-\mathbf{u}^{n}}{\Delta t}+(\mathbf{u} \cdot \nabla \mathbf{u})^{n}\right) \\
& \quad=-\nabla p^{n+1}+\nabla \cdot\left(\mu_{s}\left(\nabla \mathbf{u}^{n+1}+\left(\nabla \mathbf{u}^{n+1}\right)^{\mathrm{T}}\right)\right)+\nabla \cdot \boldsymbol{\tau}^{n}+\mathbf{F}^{n}
\end{aligned}
$$


Due to the incompressibility constraint the numerical solution of the Eqs. (16) and (17) couples the velocity and pressure fields. To overcome this difficulty the projection method is applied. The essence of the projection method is to calculate an intermediate velocity $\tilde{\mathbf{u}}$, through Eq. (17) by approximating $p^{n+1}$ by $p^{n}$, i.e.,

$$
\begin{aligned}
& \rho\left(\frac{\widetilde{\mathbf{u}}^{n+1}-\mathbf{u}^{n}}{\Delta t}+(\mathbf{u} \cdot \nabla \mathbf{u})^{n}\right) \\
& \quad=-\nabla p^{n}+\nabla \cdot\left(\mu_{s}\left(\nabla \widetilde{\mathbf{u}}^{n+1}+\left(\nabla \widetilde{\mathbf{u}}^{n+1}\right)^{\mathrm{T}}\right)\right)+\nabla \cdot \boldsymbol{\tau}^{n}+\mathbf{F}^{n} .
\end{aligned}
$$

This strategy decouples the velocity and pressure fields, but it is necessary to correct the pressure and calculate a velocity field that satisfies Eq. (16). In this case the pressure correction is achieved by putting

$p^{n+1}=p^{n}+\frac{\psi^{n+1}}{\Delta t}$

and the velocity field that satisfies Eq. (16) is obtained from

$\mathbf{u}^{n+1}=\widetilde{\mathbf{u}}^{n+1}-\frac{1}{\rho} \nabla \psi^{n+1}$,

where $\psi^{n+1}$ is calculated by the solution of the Poisson equation

$\nabla \cdot\left(\frac{1}{\rho} \nabla \psi^{n+1}\right)=\nabla \cdot \widetilde{\mathbf{u}}^{n+1}$.

\subsection{Viscoelastic constitutive equation}

After updating the velocity and pressure fields, it remains to update the non-Newtonian stress tensor by solving the viscoelastic constitutive equation. In the present work, we have implemented a standard extra-stress and a stabilized formulation. For low Weissenberg number flows, the transport equation for the non-Newtonian stress tensor is given by Eq. (3). In order to be able to simulate HWNP, the generic kernel transformation formulation is adopted, and consequently, Eq. (11) need to be solved. In summary, Eqs. (3) and (11) are discretized in time, respectively, as follows:

$$
\begin{aligned}
\frac{\boldsymbol{\tau}^{n+1}-\boldsymbol{\tau}^{n}}{\Delta t}= & -\left[\left(\mathbf{u}^{n+1} \cdot \nabla\right) \boldsymbol{\tau}^{n}\right]+\left(\nabla \mathbf{u}^{n+1}\right)^{T} \cdot \boldsymbol{\tau}^{n} \\
& +\boldsymbol{\tau}^{n} \cdot \nabla \mathbf{u}^{n+1}+\frac{1}{\lambda} \mathbf{M}\left(\boldsymbol{\tau}^{n}\right)
\end{aligned}
$$

and

$$
\begin{aligned}
\frac{\mathbb{k}(\mathbf{A})^{n+1}-\mathbb{k}(\mathbf{A})^{n}}{\Delta t}= & -\left[\left(\mathbf{u}^{n+1} \cdot \nabla\right) \mathbb{k}(\mathbf{A})^{n}\right]+\mathbf{\Omega}^{n+1} \mathbb{k}(\mathbf{A})^{n} \\
& -\mathbb{k}(\mathbf{A})^{n} \boldsymbol{\Omega}^{n+1}+2 \mathbb{B}^{n}+\frac{1}{\lambda} \mathbb{M}^{n} .
\end{aligned}
$$

Remark: Once obtained the value of $\mathbb{k}(\mathbf{A})^{(n+1)}$, the inverse kernel function is used to obtain the conformation tensor $\mathbf{A}^{n+1}$, and finally, the non-Newtonian extra-stress tensor $\boldsymbol{\tau}^{(n+1)}$ is computed from Eq. (8). More details about the numerical procedure to deal with the kernel-conformation tensor $\mathbb{k}(\mathbf{A})$ can be found in Martins et al., 2015.

The boundary conditions for the extra-stress tensor, necessary to solve Eq. (22), are the homogeneous Neumann conditions in outlets and symmetry planes. Notice that wall boundary conditions are not needed for the extra-stress tensor due to its hyperbolic nature. At the interface, the constitutive equation is solved by interpolating the properties of both phases using Eq. (13) (see more details in Khismatullin et al. (2006)). In order to maintain the second-order accuracy of discretization of Eq. (18), the divergent of the extra-stress tensor is calculated by a forward or backward second-order difference scheme in the cells neighboring the rigid wall.

\subsection{Non-uniform mesh}

Non-uniform grids, also known as stretched grids, are a popular form of adaptive meshing, where more grid points are positioned in regions of interest or where higher gradients are expected. They are particularly useful in the rod-climbing problem, since higher resolution is desired near the rod. They are very popular due to its ease of implementation. Most finite-differences codes can be easily adapted to stretched grids.

The main drawback is that central differences become firstorder accurate if adjacent points are not equally spaced. Forward and backward differences remain first-order. According to (Ferziger and Peric, 2012) the truncation error in non-uniform grids can be written as

$e_{\tau} \approx \frac{\left(1-r_{e}\right) \Delta x_{i}}{2}\left(\frac{\partial^{2} \phi}{\partial x^{2}}\right)_{i}$,

where $\Delta x_{i}=x_{i}-x_{i-1}$ and $r_{e}=\Delta x_{i+1} / \Delta x_{i}$ is close to one, firstorder errors decrease faster than second-order ones. As the grid is refined, convergence becomes asymptotically second order for central differences.

In non-uniform grids, central first derivatives become

$\left(\frac{\partial \phi}{\partial x}\right)_{i}=\frac{\phi_{i+1}\left(\Delta x_{i}\right)^{2}-\phi_{i-1}\left(\Delta x_{i+1}\right)^{2}+\phi_{i}\left[\left(\Delta x_{i+1}\right)^{2}-\left(\Delta x_{i}\right)^{2}\right]}{\Delta x_{i+1} \Delta x_{i}\left(\Delta x_{i+1}+\Delta x_{i}\right)}$

and central second derivatives become

$\left(\frac{\partial^{2} \phi}{\partial x^{2}}\right)_{i}=\frac{\phi_{i+1} \Delta x_{i}+\phi_{i-1} \Delta x_{i+1}-\phi_{i}\left(\Delta x_{i+1}+\Delta x_{i}\right)}{\frac{1}{2}\left(\Delta x_{i+1}+\Delta x_{i}\right) \Delta x_{i+1} \Delta x_{i}}$.

The mesh generation is one of the difficulties encountered in solving fluid flow. In this work we have adopted a structured mesh with MAC type cells Figueiredo et al., 2013. Thus we can generate a non-uniform mesh stretching or compressing the cells in each direction separately. We do this by using grid control function to get the points $x_{i}$ in the domain. Two different grid control functions were employed in this work. We used a geometric progression, defined as

$x_{i}=r_{e} x_{i-1}$,

where $r_{e}$ is the progression ratio. We can also use a different nonuniform mapping, defined as

$x_{i}=L \bar{x}_{i}^{a}+A\left(x_{c}-L \bar{x}_{i}\right)\left(1-\bar{x}_{i}\right) \bar{x}_{i}$

where $\bar{x}_{i} \in[0,1]$ is a uniform distribution of points, $L$ is the width of the computational domain, $A$ and $a$ are compression/expansion geometrical factors and $x_{c}$ is a point of attraction of the grid lines.

\subsection{Computational algorithm}

The numerical procedure used to approximate the solution of all variables at a time step, $t=t^{n}+\delta t$, consists in the following steps:

(1) The first step is to treat the interface representation solving the advection equation Eq. (12) through a split operator with a geometric procedure;

(2) Reconstruct the interface using the ELVIRA method;

(3) The next step is the calculation of the surface tension term (14). The curvature is calculated by the HF method;

(4) Calculate the intermediate velocity field, $\widetilde{\mathbf{u}}$, solving Eq. (18);

(5) The next step is to apply the projection method, which calculates the pressure correction by solving the Poisson equation, Eq. (21). After computing the pressure correction, the velocity and the pressure fields are updated by Eqs. (19) and (20); 
(6) The last step is to obtain the non-Newtonian tensor by solving Eq. (22). If the flow has a high Weissenberg number, the constitutive equation is solved using stabilization methods, Eq. (23);

\section{Numerical validations}

In this Section, we present the numerical validation of the new viscoelastic two-phase solver, in order to assess the numerical implementation accuracy for distinct levels of physical complexities. First, a confined problem with single-phase fluid is used a benchmark problem to assess both the implementation of the NavierStokes and constitutive equations (in both standard and matrix transformation formulations) in 2D Cartesian coordinates (the viscoelastic laminar lid-driven cavity flow - see Section 4.1) and in 1D a 2D axisymmetric coordinates (the concentric annulus with inner cylinder rotation - see Section 4.2). Finally, to assess the validation of the numerical implementation for a $2 \mathrm{D}$ axisymmetric flow with two-phases, we study two benchmark test problems from the literature, namely the shear deformation of a droplet, in Section 4.3, and the impacting drop problem - see Section 4.4. In order to maintain the accuracy and stability of the code, we have used moderate density and viscosity ratios. In particular, the maximum ratios used in this work are: $\frac{\rho_{\text {fluid }}}{\rho_{\text {outer }}} \leq 816.33$ and $\frac{\mu_{\text {fluid }}}{\mu_{\text {outer }}} \leq 200$.

4.1. Viscoelastic single-phase flow in Cartesian coordinates: lid-driven cavity problem at high Weissenberg number

In this test, we present the validation of the numerical implementations for a two-dimensional confined flow, namely the liddriven cavity benchmark flow problem. We examine and validate the accuracy of the estimation for the extra-stress terms arising from the viscoelastic constitutive equations in two dimensional Cartesian coordinates. In this Section, we present the results obtained for this problem using the Oldroyd-B fluid, and compare with data from the literature.

This benchmark has been used to verify the stabilization methods for HWNP (Fattal and Kupferman, 2005; Pan et al., 2009; Su et al., 2013; Habla et al., 2014). In particular, for $W i \geq 1$ and the Oldroyd-B model, results have been published by Fattal and Kupferman (2005) and for several Kernel function by Martins et al. (2015) and Palhares-Junior et al. (2016). In Martins et al., 2015, the authors used a Marker-And-Cell discretization implemented in the scope of finite differences method framework, while in the work of Palhares-Junior et al. (2016) the results were also obtained using a finite volume method.

The usual standard problem relies on the following regularized parabolic profile for the top lid $u(x, t)=8[1+\tanh (8 t-4)] x^{2}(1-$ $x)^{2}$. The remaining cavity walls are stationary and the no-slip boundary condition is imposed everywhere. We have fixed the Reynolds number $R e=0.01$ and the solvent viscosity ratio as $\beta=$ 0.5 . In order to show qualitative comparison with the literature, we have adopted $W i=2$.

We used a grid with cells concentrated near the walls. The nonuniform stretching of the mesh was obtained using Eq. (28), with $A=0$ and $a=0.3$ for the compression of the center of the domain to the walls. To assess the mesh convergence, the cavity flow was simulated using three non-uniform meshes: $M 1$ with $64 \times 64$ cells $\left(\min \left(\Delta x_{i}, \Delta y_{j}\right)=0.007068, i, j=1,2, \ldots, 64\right)$, M2 with $128 \times 128$ cells $\left(\min \left(\Delta x_{i}, \Delta y_{j}\right)=0.002892, i, j=1,2, \ldots, 128\right)$ and $M 3$ with $256 \times 256$ cells $\left(\min \left(\Delta x_{i}, \Delta y_{j}\right)=0.001179, i, j=1,2, \ldots, 256\right)$. For all figures in this Section, the profiles of the $u$-velocity and of the non-Newtonian $\tau_{x x}$ component are plotted along the vertical line $x=0.5$ while the $v$-velocity component is reported at the horizontal line $y=0.75$. The origin of the Cartesian coordinate system is placed at the lower left corner of the square cavity, and the moving wall is located at $y=1$. In these simulations, the time step used in the simulation was fixed as $\delta t=0.0001$ for all meshes. In order to assess the numerical stability properties of the solver at higher Weissenberg number, we have adopted the generic kernel transformation framework where the functional kernel relation is based in the logarithm of base exp.

In Fig. 1, we present a mesh refinement study and compare with literature data for the velocity fields. From Fig. 1a and b, we can observe that the results for the velocity field are in good agreement with those found by Fattal and Kupferman (2005) and Martins et al. (2015). Results for a mesh refinement study of the $x$ component of the non-Newtonian stress tensor, $\tau_{x x}$, are presented in Fig. 1c where it can be observed the numerical convergence of the solutions. In addition, in Fig. 1c, we have included the finite difference results from Martins et al. (2015) showing reasonable agreement between the numerical solutions. Finally, the time evolution of the kinetic energy obtained for meshes $M 1, M 2$, and $M 3$ is compared against the results of Martins et al. (2015), as depicted in Fig. 1d. We can observe from this figure that our results tend to a steady state showing a peak of the dimensionless kinetic energy at $E \approx 0.018$ for $t \approx 0.8$, followed by a decrease towards an asymptotic value of $E \approx 0.0093$ in M3 mesh.

\subsection{Viscoelastic single-phase flow in axisymmetric coordinates: concentric annulus with inner cylinder rotation}

Here we present the validation of the numerical implementations for an axisymmetric confined flow, namely the flow in a concentric annulus with inner cylinder rotation. This type of annular flows of non-Newtonian fluids can be found in a wide variety of applications, such as in industrial processes involving waste fluids and synthetic fibres, and on drilling for oil and gas wells, as reviewed in an extensive bibliographic list by Escudier et al. (2002). Solutions for other rheological constitutive models were presented by other authors, such as the work of Pinho and Oliveira (2000), that presented an analytical solution for the concentric annular laminar flow without inner cylinder rotation for the simplified PTT model and Mirzazadeh et al. (2005), that presented an approximate analytical solution for purely tangential flow of a viscoelastic fluid obeying the LPTT constitutive equation in a concentric annulus with relative rotation of the inner and outer cylinders. In Mirzazadeh et al. (2005), an explicit analytical solution was obtained for the the simplified PTT model. Ravanchi et al. (2007) presented an approximate analytical solution for the steady state, purely tangential flow of a viscoelastic fluid obeying the Giesekus constitutive equation in a concentric annulus with inner cylinder rotation.

In the solution of Ravanchi et al. (2007), the analytical dimensionless wall shear stress on the inner cylinder, denoted as $\tau_{W i}^{*}$, is given by a nonlinear equation. In the present paper, we have numerically solved this equation to compare with $\tau_{W i}^{*}$ obtained from the numerical method by the following equation:

$\tau_{r \theta}^{*}=\frac{\zeta^{2} \tau_{W i}^{*}}{r^{*}}$

where $\zeta$ is the radius ratio and $r^{*}$ is the non-dimensional radial coordinate. In this problem, the Weissenberg number is defined as $W i=\frac{\lambda U}{\delta}$, where $\delta$ is the annular gap.

The mesh adopted for the computations was generated with stretching in the $r$ direction from Eq. (27) with $r_{e}=1.04$ and $\Delta r_{0} / \delta \approx 2.6587 \times 10^{-4}$. The mobility factor for the Giesekus model, $\alpha$, was fixed at 0.2 in all simulations. According to Ravanchi et al. (2007), the approximate solution is valid for small values of $4 \alpha^{2} W i^{2} \tau_{r \theta}^{*^{2}}$, and thus increasing the value of the mobility factor, $\alpha$, the range of acceptable Weissenberg numbers become narrower. Boundary conditions are imposed as follows: in the inner cylinder, 

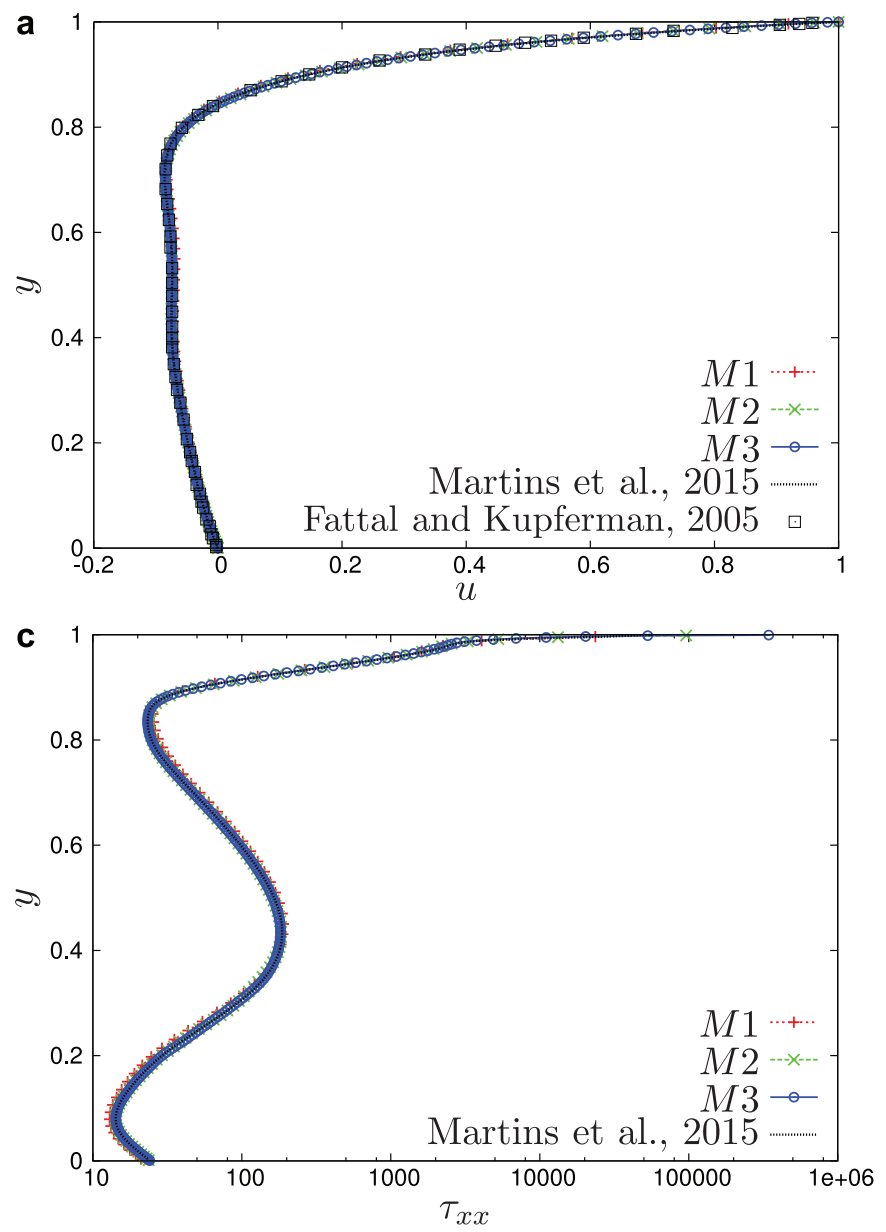
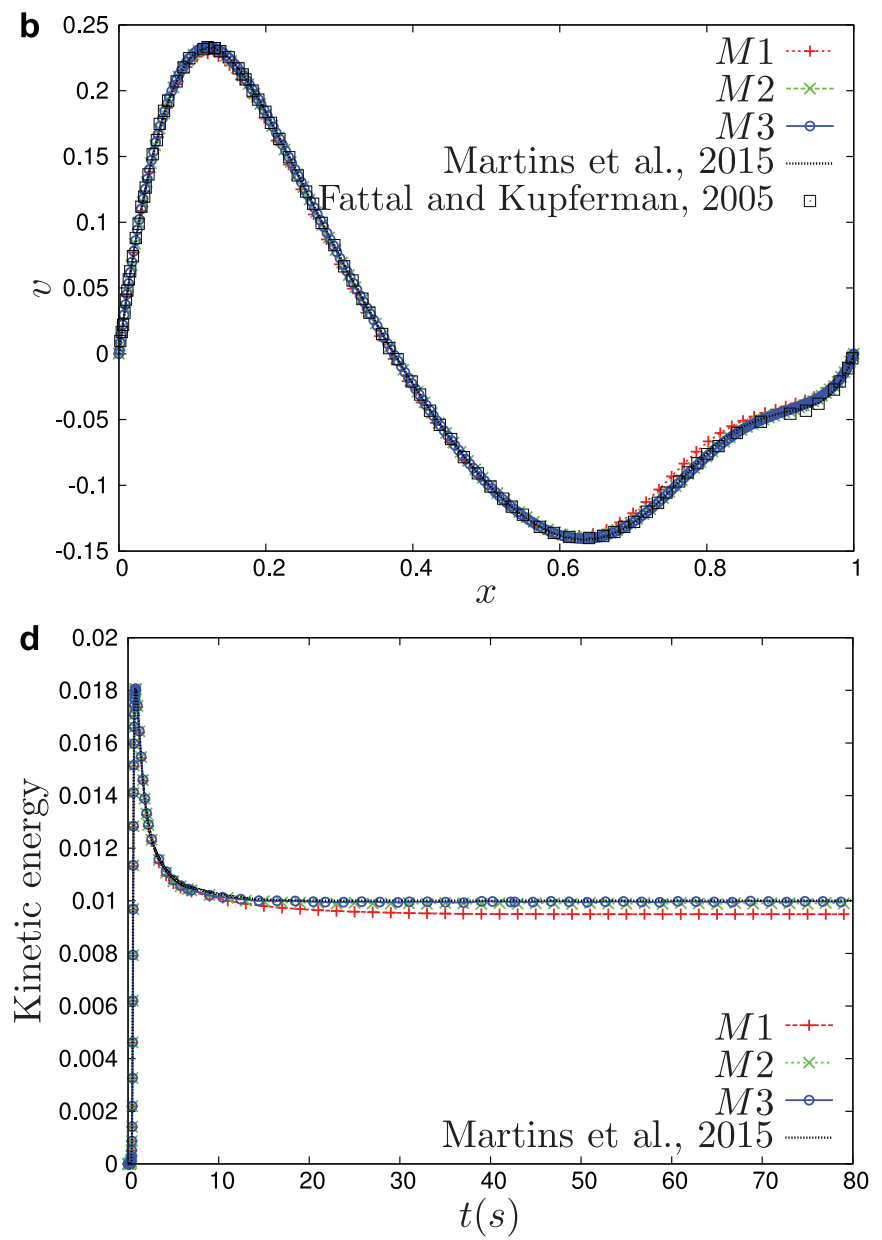

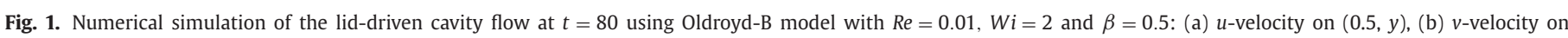
$(x, 0.75),(c) \tau_{x x}$ on $(0.5, y)$, and (d) kinetic energy.

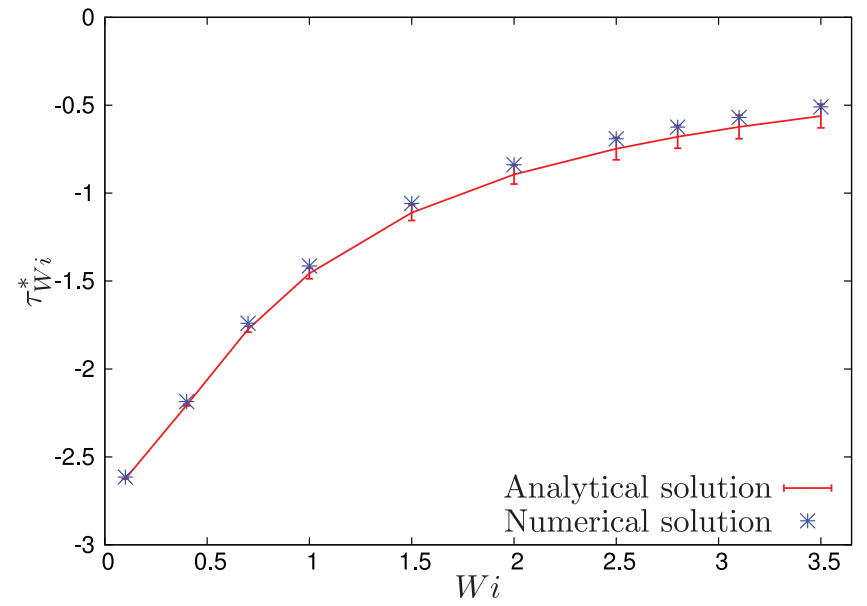

Fig. 2. Dimensionless wall shear stress $\tau_{W i}^{*}$ on the inner cylinder predicted numerically and by the approximate analytical solution of Ravanchi et al. (2007). The error bars represent the percentage of truncation error introduced in Ravanchi et al., 2007.

a uniform rotating velocity $V_{c}$ is imposed while no-slip boundary condition is adopted in the outer cylinder.

Fig. 2 presents the dimensionless wall shear stress $\tau_{W i}^{*}$ on the inner cylinder predicted numerically and by the approximate analytical solution of Ravanchi et al. (2007). As we can observe, the percentage of truncation error introduced by the approximate so- lution increases when the Weissenberg numbers increases. This fact is in agreement with the studies presented by Ravanchi et al. (2007).

Finally, we show in Fig. 3 the dimensionless velocity and stress profiles obtained for several Weissenberg numbers, which are in good agreement with the analytical approximate solutions, within the respective truncation error.

\subsection{Viscoelastic two-phase flow in Cartesian coordinates: deformation droplet}

In this section we validate the numerical implementations for a viscoelastic two-phase flow, using Cartesian coordinates. In this test, we study the behavior of a deformable droplet under a pure Couette shear flow, in which either or both the droplet and bulk fluid may be considered as a viscoelastic fluid. All four possible combinations for the droplet-bulk fluid system are considered in the present work: (i) a Newtonian droplet in a Newtonian fluid (NN) (ii) a viscoelastic droplet in a Newtonian fluid (VN) (iii) a Newtonian droplet in a viscoelastic fluid (NV) and (iv) a viscoelastic droplet in a viscoelastic fluid (VV). Similar tests were used by Pillapakkam and Singh (2001) using a Level-Set method, Habla et al. (2011) using a VOF method with conditionally volume averaging, Chinyoka et al. (2005) and Khismatullin et al. (2006), using an 2D and 3D/PROST algorithm based on the VOF method, respectively.

The initial configuration used for the simulation of transient droplet deformation subjected to a steady Couette planar 


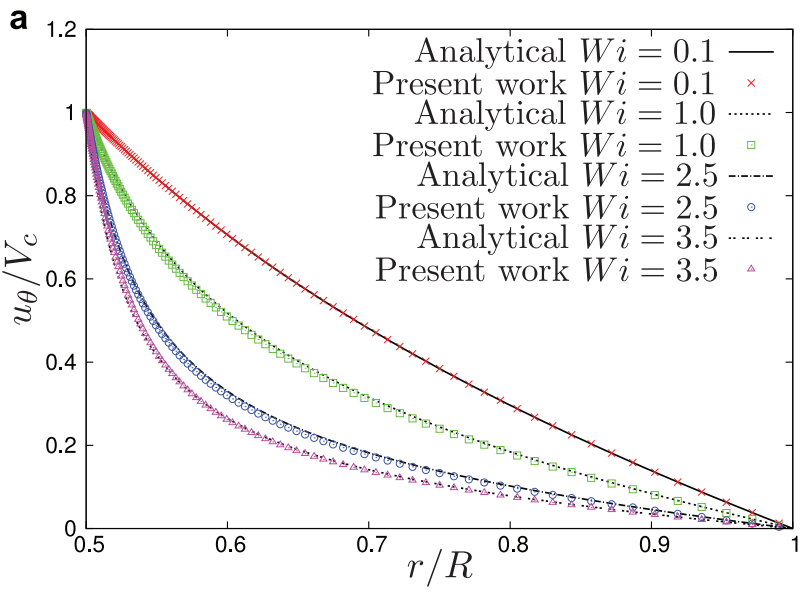

C

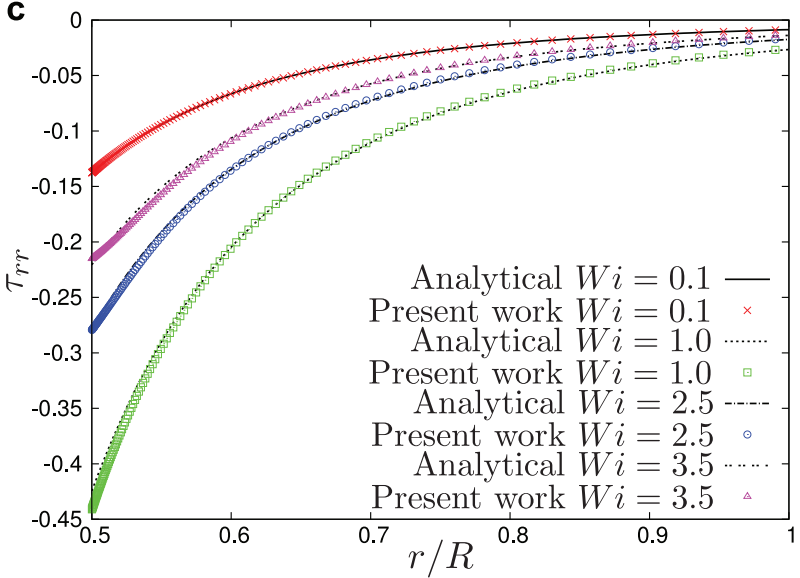

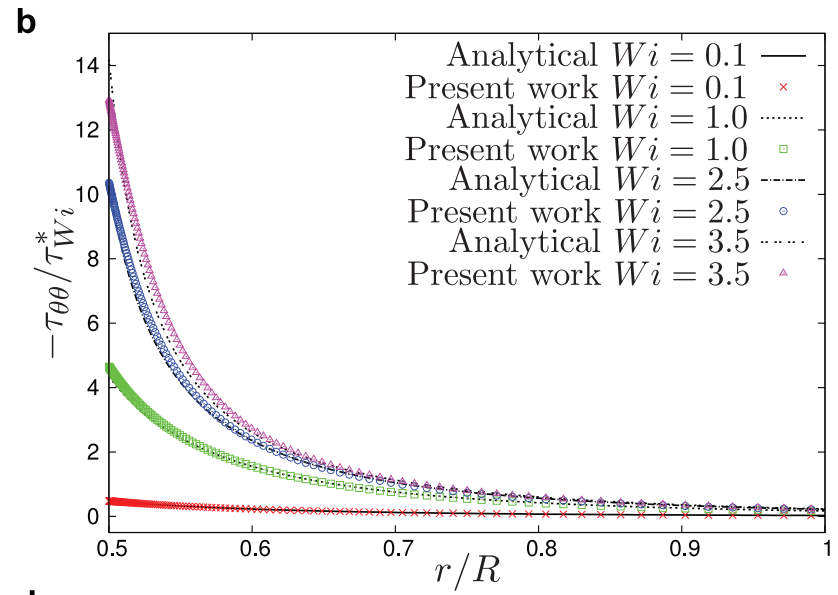

d

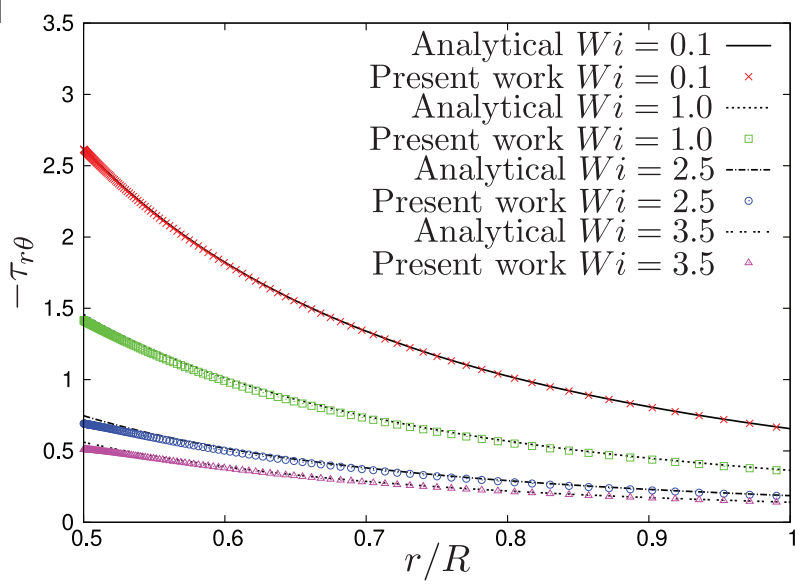

Fig. 3. Numerical simulation for the concentric annulus with inner cylinder rotation using the Giesekus model $\alpha=0.2$ : (a) $u_{\theta}$-velocity, (b) $-\tau_{\theta \theta}$, (c) $\tau_{r r}$ and (d) $-\tau_{r \theta}$.
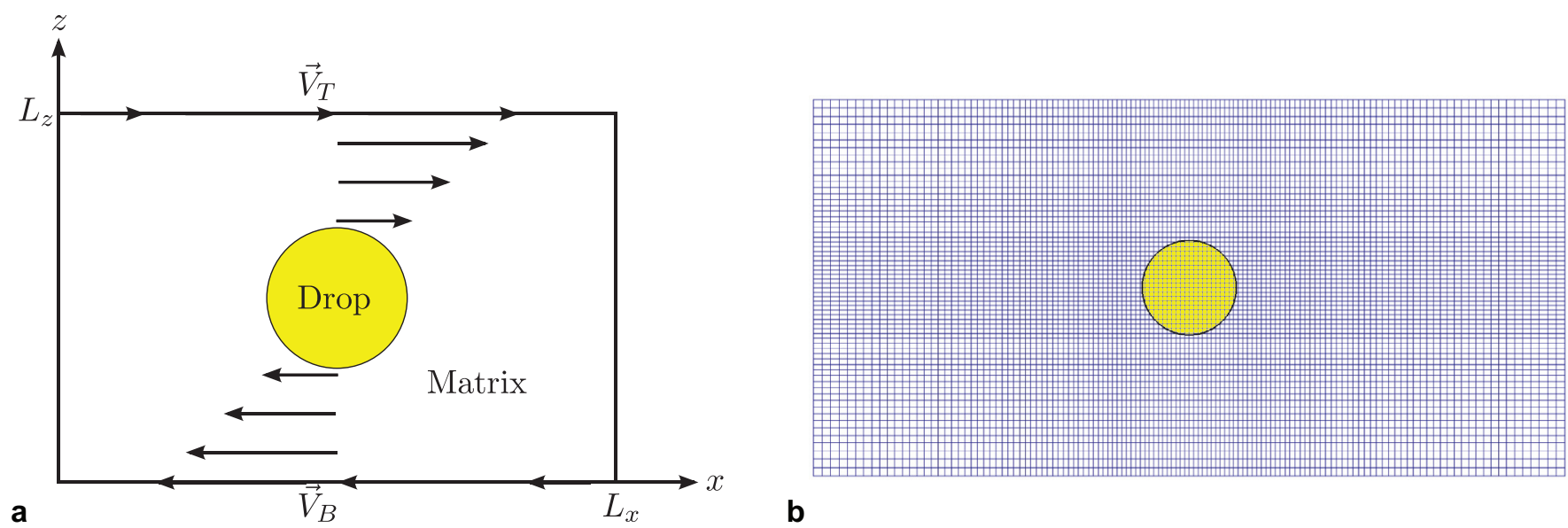

b

Fig. 4. Initial configuration for the deformation droplet flow: (a) sketch view of the numerical experiments and (b) non-uniform mesh representation.

shear-flow is presented in Fig. 4a. An initially circular droplet of radius $R_{d}$ is located at the center of a rectangular domain and subjected to a simple Couette shear flow, with the bottom and top rigid walls moving in opposite directions, with velocities $V_{B}$ and $V_{T}$, respectively. The bottom wall is located at $z=$ 0 , while the top wall is located at $z=L_{z}=8 R_{d}$. The horizontal channel length is $L_{X}$ and is taken large enough compared with the initial drop radius, so that the drop is effectively at constant shear rate, $\dot{\gamma}=\left(V_{T}-V_{B}\right) / L_{z}$. Notice that in the lateral boundaries we have used homogeneous Neumann boundary condition for all fields.
Here the viscoelastic fluid is defined as an Oldroyd-B liquid, in which the amount of Newtonian solvent is controlled by the dimensionless solvent viscosity coefficient, $\beta$. When , $\beta=1$ the fluid becomes Newtonian while a value close to zero corresponds to a more elastic fluid. The other dimensionless parameters used in this test case are defined as: $C a=\frac{\mu_{0} \dot{\gamma} R_{d}}{\sigma}, R e=\frac{\rho \dot{\gamma} R_{d}^{2}}{\mu_{0}}$ and $W i=\lambda \dot{\gamma}$. The deformation parameter, $\Phi$, is defined as the ratio between the smaller and larger distances of the droplet interface up to the drop center, as $\Phi=\left(R_{\max }-R_{\min }\right) /\left(R_{\max }+R_{\min }\right)$.

The domain was discretized by two different non-uniform meshes: $\quad M 1 \quad\left(\min \left(\Delta x_{\min }, \Delta y_{\min }\right) / R_{d} \approx 4.6876 \times 10^{-2}, 256 \times 128\right.$ 

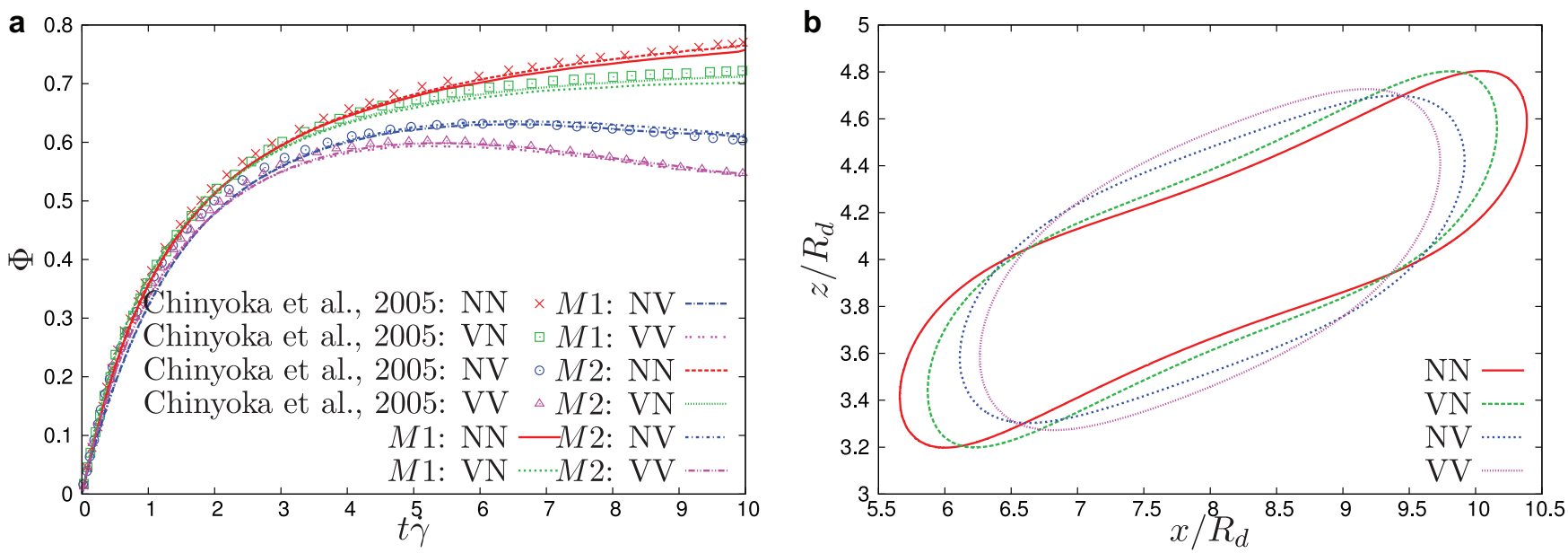

Fig. 5. (a) Time evolution for the deformation parameter, $\Phi$, for all droplet-bulk fluid configurations: NN, VN, NV and ,VV; (b) droplet shapes at $t=10 \dot{\gamma}^{-1}$.

cells) and $M 2$ with $\left(\min \left(\Delta x_{\min }, \Delta y_{\min }\right) / R_{d} \approx 2.3438 \times 10^{-2}, 512 \times\right.$ 256 cells). These meshes were generated using the Eq. (28), with $A=1, a=1$ and $x_{c}=L_{X} / 2$, as observed in Fig. 4b. The time step size is determined so as to satisfy the CFL conditions due to surface tension and viscous terms.

Fig. 5 a shows the results for $C a=0.6, R e=0.3, \beta=0.5$ and $W i=0.4$, the same parameters used by Chinyoka et al. (2005). The obtained results are in good agreement with literature data. We can observe that when the outer fluid is Newtonian the droplet deforms continuously along time, while it settles to a stationary state when the outer fluid is viscoelastic. The interface shape of the deformed droplet at $t=10 \dot{\gamma}^{-1}$ is presented in Fig. 5b, showing that when the viscoelastic droplet in the VN system presents a dumbbell shape whereas the Newtonian droplet in the NV system has an almost elliptical shape.

\subsection{Viscoelastic two-phase flow in axisymmetric coordinates: impacting drop problem}

Finally, by this problem, we present the validation of the numerical implementations for an axisymmetric flow with twophases. In the impacting drop problem, we compute the time evolution of the shape of a drop that falls under gravity action from a distance $H$ above a rigid wall. In this Section, we present the results obtained for this problem using the Oldroyd-B fluid, and compare with data from the literature (Xu et al., 2012; Figueiredo et al., 2014). Fig. 6 illustrates the initial drop shape used in the transient motion.

The parameters used in this study were: $D=0.02 \mathrm{~m}, U=1 \mathrm{~m} / \mathrm{s}$ and $g=-9.81 \mathrm{~m} / \mathrm{s}^{2}$ (resulting in $F r=2.26$ ). We consider a drop with an initial velocity $v_{z}=-1 \mathrm{~m} / \mathrm{s}$ at a distance $H=0.04 \mathrm{~m}$ from the center of the drop to the rigid wall. To verify the implementation of the code, we simulate the problem of the Oldroyd-B fluid model adopting: $\operatorname{Re}=5.0, \mathrm{Fr}=2.26, W i=1.0$ and $\beta=0.1$. The drop is falling into a Newtonian fluid phase with $\rho \rightarrow 0$ and $\mu$ $\rightarrow 0$. We have imposed symmetry boundary conditions on the left while no-slip boundary condition is used in the rigid wall located at the bottom.

Three meshes were used for the computations: $M 1$ with $\Delta r=$ $\Delta z=5 \times 10^{-4}, M 2$ with $\Delta r=\Delta z=3.33 \times 10^{-4}$ and $M 3$ with $\Delta r=$ $\Delta z=2.5 \times 10^{-4}$. Fig. 7 shows the comparison among our results and those in the literature. The results obtained for the three meshes show good convergence with mesh refinement, and in comparison with the other methods, our code produces similar qualitative results. In Fig. 8 we present a $3 \mathrm{D}$ view of the droplet spreading time evolution for $W i=1.0$ and $\beta=0.1$.

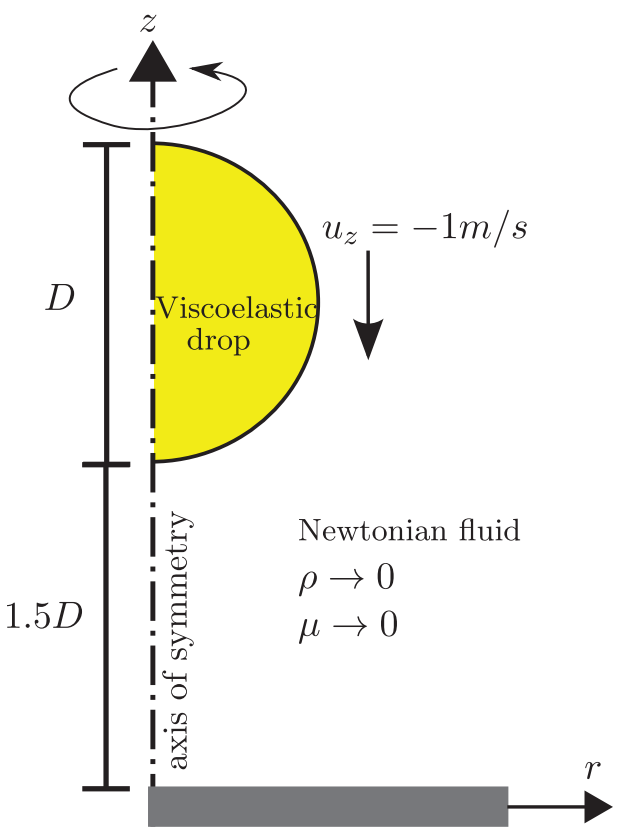

Fig. 6. Sketch view of the numerical experiments for the impacting drop problem.

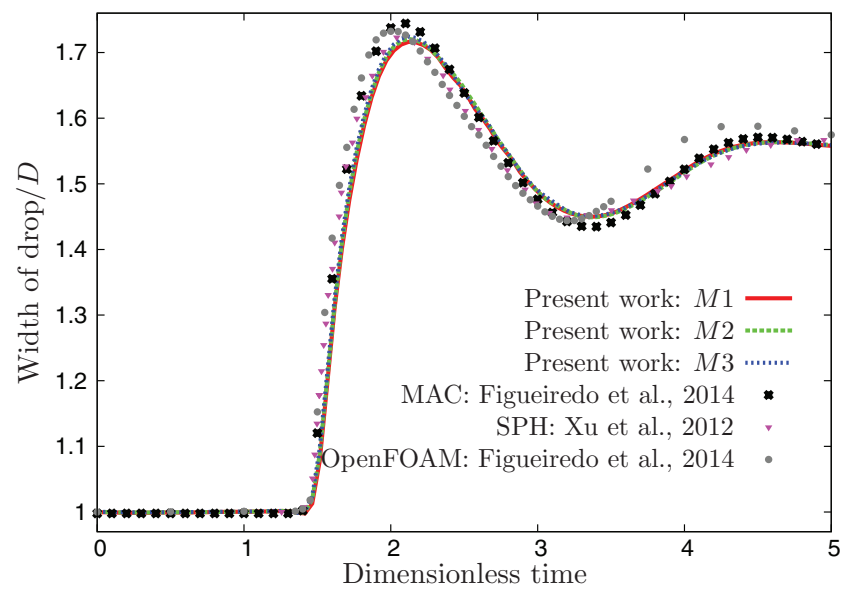

Fig. 7. Numerical prediction of the time variation of the width of viscoelastic drop. 

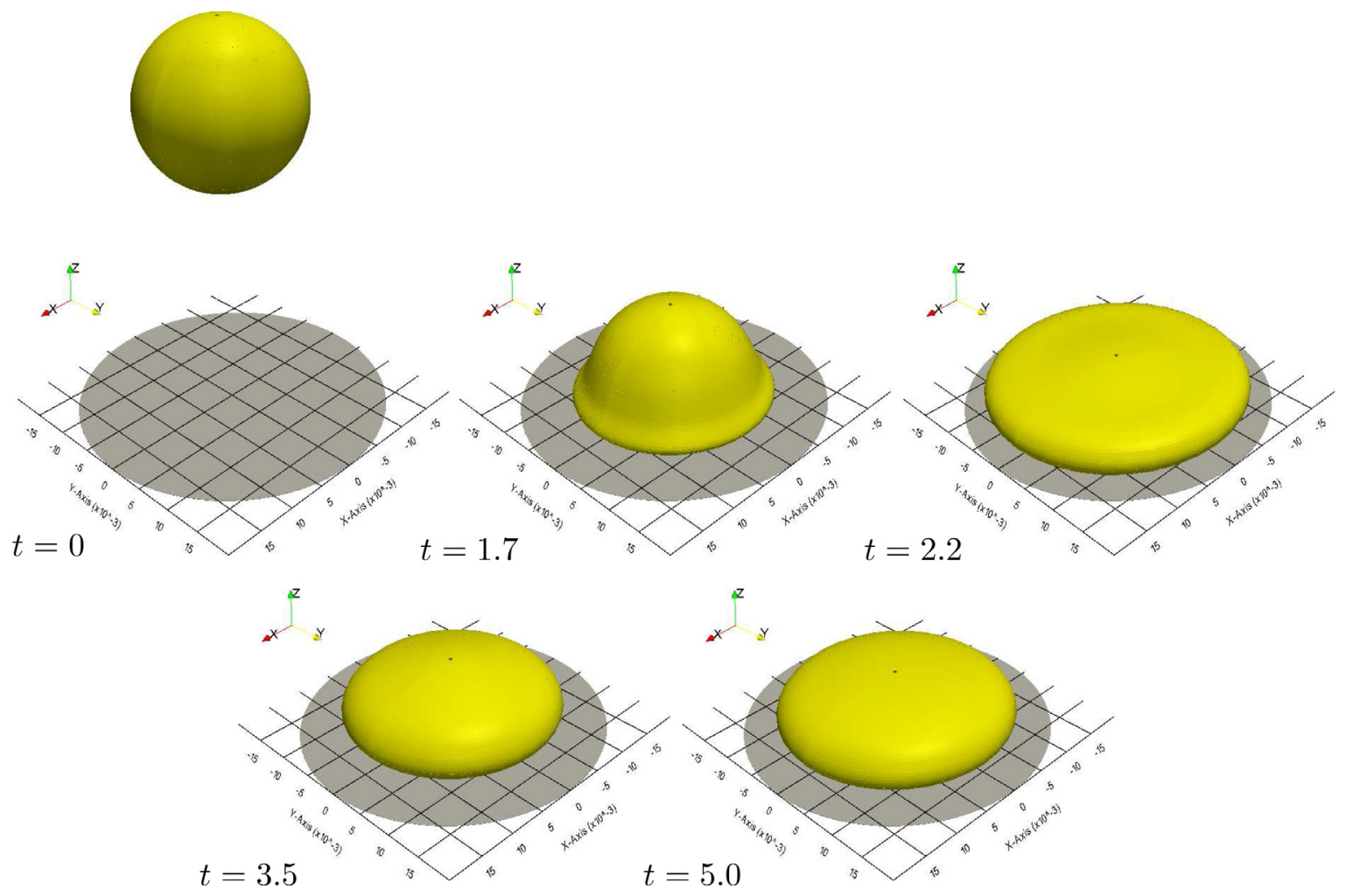

Fig. 8. 3D view of droplet spreading time evolution obtained for $W i=1.0$ and $\beta=0.1$.

\section{Application case: the Weissenberg effect}

The Weissenberg effect is an interesting problem in Fluid Mechanics for which many peculiar behaviors can be observed. A major contributor to these studies was compiled by Joseph and his co-workers in Joseph, 1990. Several authors performed experimental and theoretical predictions of the Weissenberg effect (Beavers and Joseph, 1975; Beavers et al., 1980; Joseph et al., 1984). On the other hand, fewer studies can be found considering numerical simulations of this effect. Debbaut and Hocq, 1992 presented numerical results for a finite element method and axisymmetric coordinates. In their study secondary flows were analyzed for the Weissenberg effect problem and results were compared with experimental and analytical solutions. Also, using FEM and axisymmetric coordinates, Luo (1999) presented numerical results for the Upper convected Maxwell and Phan-Thien-Tanner models. In addition, Luo (1999) proposed an elaborate scheme of orthogonal trajectories to update structured and unstructured meshes, showing good agreement between numerical and experimental results. Recently, Habla et al. (2011) developed an algorithm for solving viscoelastic two phase flows in the OpenFOAM code. In Habla et al., 2011 numerical results of the Weissenberg effect were obtained with the VOF method where the interface was represented in a three-dimensional framework.

The Weissenberg effect or rod climbing occurs due to the influence of normal stress differences in the variation of the pressure value in the radial direction (Bird et al., 1987). Therefore, the normal stresses ( $N 1$ and $N 2$ ), may cause the total normal pressure to decrease in the radial direction, as observed by the following relation (Bird et al., 1987):

$$
\frac{\partial\left(\tau_{z z}+p\right)}{\partial \ln r}=2 \tau_{r \theta} \frac{\partial}{\partial \tau_{r \theta}} \underbrace{\left(\tau_{r r}-\tau_{z z}\right)}_{N_{2}}+\underbrace{\left(\tau_{\theta \theta}-\tau_{r r}\right)}_{N_{1}}+\rho u_{\theta}^{2}
$$

In this Section, two viscoelastic models were adopted to simulate the Weissenberg effect: the Oldroyd-B model and a submodel of the linear Phan-Thien-Tanner (Phan-Thien and Tanner, 1977) with $\epsilon=0$, called the Johnson-Segalman model Johnson and Segalman (1977). Note that as $\xi$ vanishes, one recovers the Oldroyd-B model. The Johnson-Segalman model satisfies the second normal stress differences, which is also responsible for the climbing effect of the fluid (Bird et al., 1987).

The geometry of the problem consists of a rod of radius $R$ rotating at a constant angular velocity $\omega$, inserted into a cylindrical container of radius $R_{c}$ and height $H$ filled with a viscoelastic fluid, as displayed in Fig. 9. The other phase was considered as a Newtonian fluid, with $\rho=1.2 \times 10^{-3} \mathrm{~g} / \mathrm{cm}^{3}$ and $\mu_{s}=1.81 \mathrm{~g} /(\mathrm{cm} \cdot \mathrm{s})$. The geometric parameters are $R=0.635 \mathrm{~cm}, R_{C} \approx 24 R$ and $H \approx 12 R$. We have imposed a uniform angular velocity $\omega$ on the rod and noslip boundary conditions at the bottom and right boundaries. Due to the geometry of the Weissenberg effect problem, which is usually composed of a rod and a container both of cylindrical form, it is convenient to use a cylindrical coordinate system for the mathematical model. The climbing height of the fluid is measured by $h / R=(\tilde{h}(r)-H) / R$, where $\tilde{h}(r)$ is the height of the interface.

\subsection{Mesh refinement study}

The system of governing equations is solved using a mesh generated using a geometric progression - see Eq. (27). The stretching factor $r_{e}=1$ was used in the $r$ direction from the rod with length $2 R$ and in the $z$ direction near the interface with length $3 R$. To expand the cells away from the near rod/interface region of the computational domain, an expansion ratio was adopted as $r_{e}=1.07$ (see Fig. 9b)).

A study of mesh refinement was performed with the following meshes: $M 1$ with $\Delta r_{\min }=\Delta z_{\min }=0.125$, M2 with $\Delta r_{\text {min }}=$ $\Delta z_{\min }=0.0625$, M3 with $\Delta r_{\min }=\Delta z_{\min }=0.03125$ and $M 4$ with $\Delta r_{\min }=\Delta z_{\min }=0.015625$. This mesh refinement study was 


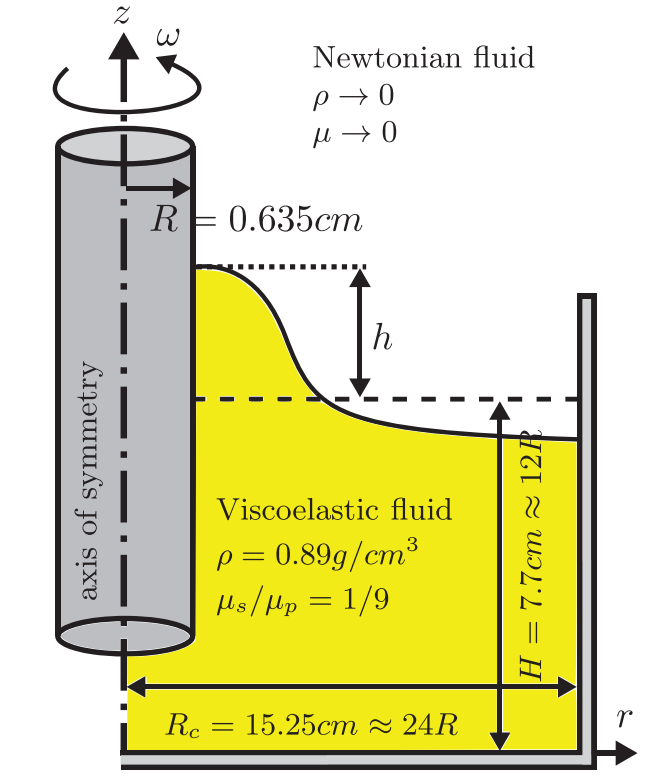

a

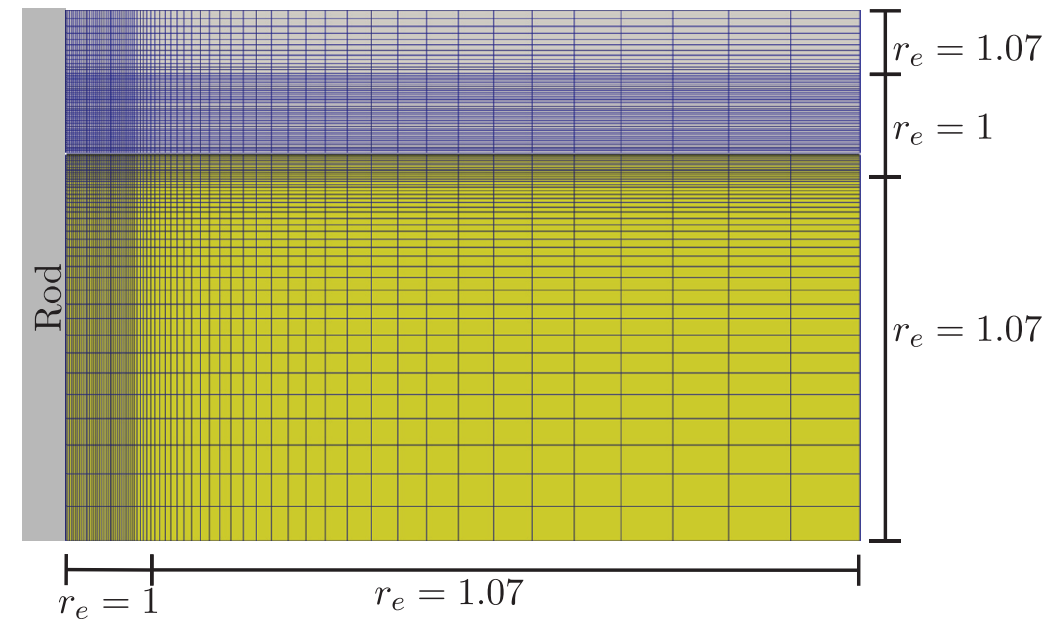

Fig. 9. The Weissenberg effect (rod-climbing problem): (a) sketch view of the numerical experiments and (b) non-uniform mesh representation.

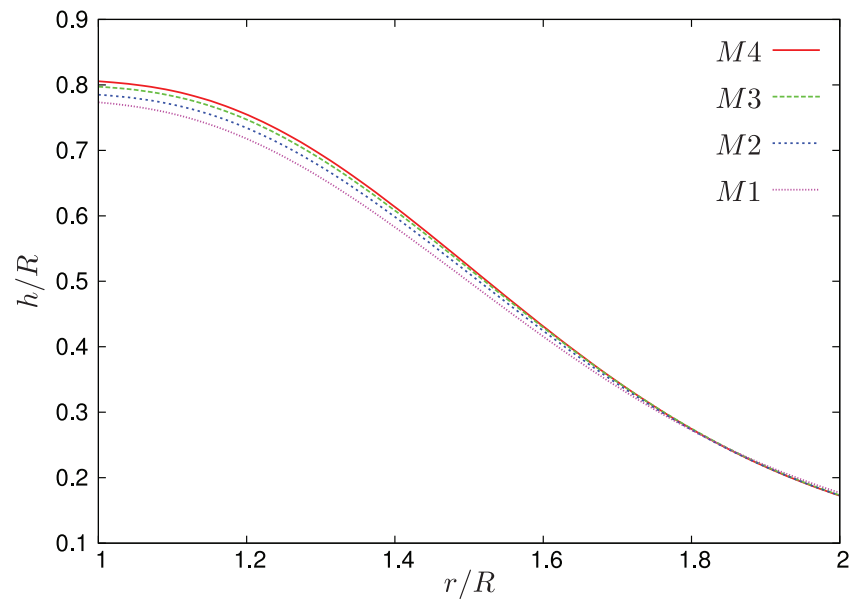

Fig. 10. Mesh refinement study for the Weissenberg effect using the Oldroyd-B model $(\xi=0)$ with $\omega=2.6 \mathrm{rev} / \mathrm{s}$.

performed for an angular velocity of $\omega=2.6 \mathrm{rev} / \mathrm{s}$ using $\xi=0$, i.e., the Oldroyd-B model. Fig. 10 shows the numerical values for the climbing height as function of the dimensionless minimum cell spacement. We can observe numerical convergence for this problem.

\subsection{Experimental and numerical comparisons}

Beavers and Joseph (1975) reported theoretical predictions on rod climbing for a fourth-order Rivlin-Ericksen fluid, claiming that their predictions showed good quantitative agreement with experimental measurements made with a STP fluid Beavers and Joseph (1975), for low values of angular velocity. Also for low angular velocity, Beavers and Joseph (1975) showed that the climbing height $h$ can be predicted analytically by

$h=\frac{R}{2 \sigma \sqrt{\frac{\rho g}{\sigma}}}\left[\frac{4 \hat{\beta}}{4+R \sqrt{\frac{\rho g}{\sigma}}}-\frac{\rho R^{2}}{2+R \sqrt{\frac{\rho g}{\sigma}}}\right] \frac{W i^{2}}{\lambda^{2}}+\mathcal{O}\left(W i^{4}\right)$
Table 1

Angular velocity and dimensionless parameter group used to verify the proposed methodology.

\begin{tabular}{lllll}
\hline & Case 1 & Case 2 & Case 3 & Case 4 \\
\hline$\omega($ rev/s $)$ & 1.7 & 2.1 & 2.6 & 2.9 \\
$W i=\lambda \omega$ & 0.195 & 0.240 & 0.298 & 0.332 \\
$R e=\frac{\rho \omega R^{2}}{\mu}$ & 0.026 & 0.032 & 0.040 & 0.045 \\
$S t=\frac{\mu \omega}{\rho g R}$ & 2.813 & 3.475 & 4.302 & 4.798 \\
$C a=\frac{\mu \omega R}{\sigma}$ & 32.05 & 39.59 & 49.01 & 54.67 \\
\hline
\end{tabular}

where the climbing constant, $\hat{\beta}$, defined as

$\hat{\beta}=\left[\left(1-\frac{\mu_{s}}{\mu_{p}}\right)\left(\mu_{s}+\mu_{p}\right) \lambda\right](1-2 \xi)$

is a linear combination of the first and second normal stress coefficients. Eq. (30) predicts a quadratic dependence of the climbing $h$ upon Wi.

To verify the proposed methodology, we perform a set of simulations for the same experimental geometry described in Beavers and Joseph, 1975, varying the angular velocity. In order to compare our results with those presented in the literature (Beavers and Joseph, 1975; Debbaut and Hocq, 1992; Luo, 1999; Habla et al., 2011), the following parameters were employed: $\rho=$ $0.89 \mathrm{~g} / \mathrm{cm}^{3}, \mu=\mu_{s}+\mu_{p}=146$ poise, $\mu_{s} / \mu_{p}=1 / 9, \lambda=0.0162(1-$ $\left.\frac{\mu_{s}}{\mu_{p}}\right)^{-1} \mathrm{~s}, \sigma=30.9 \mathrm{dyn} / \mathrm{cm}, g=981 \mathrm{~cm} / \mathrm{s}^{2}$ and $\epsilon=0$. Moreover, in the Weissenberg effect problem there are four dimensionless parameters that relate the properties of the fluid with the geometric parameters of the flow, for instance, $\mathrm{Wi}, \mathrm{Re}, \mathrm{St}$ and $\mathrm{Ca}$, introduced in Section 2 and defined for this problem in Table 1. Experimentally, Beavers and Joseph (1975) used a coated rod, so that at the contact point the free surface is normal to the rotating rod. In the present simulation, we impose that the two-fluid interface has a vanishing curvature on the rod.

The parameter $\xi$ is determined by the climbing constant $\hat{\beta}$, which depends on the temperature that can obtained from experimental data. According to Beavers and Joseph, 1975 and Luo, 1999, at temperature $27^{\circ} \mathrm{C}$ this constant has the value $\hat{\beta}=0.86$ and at 

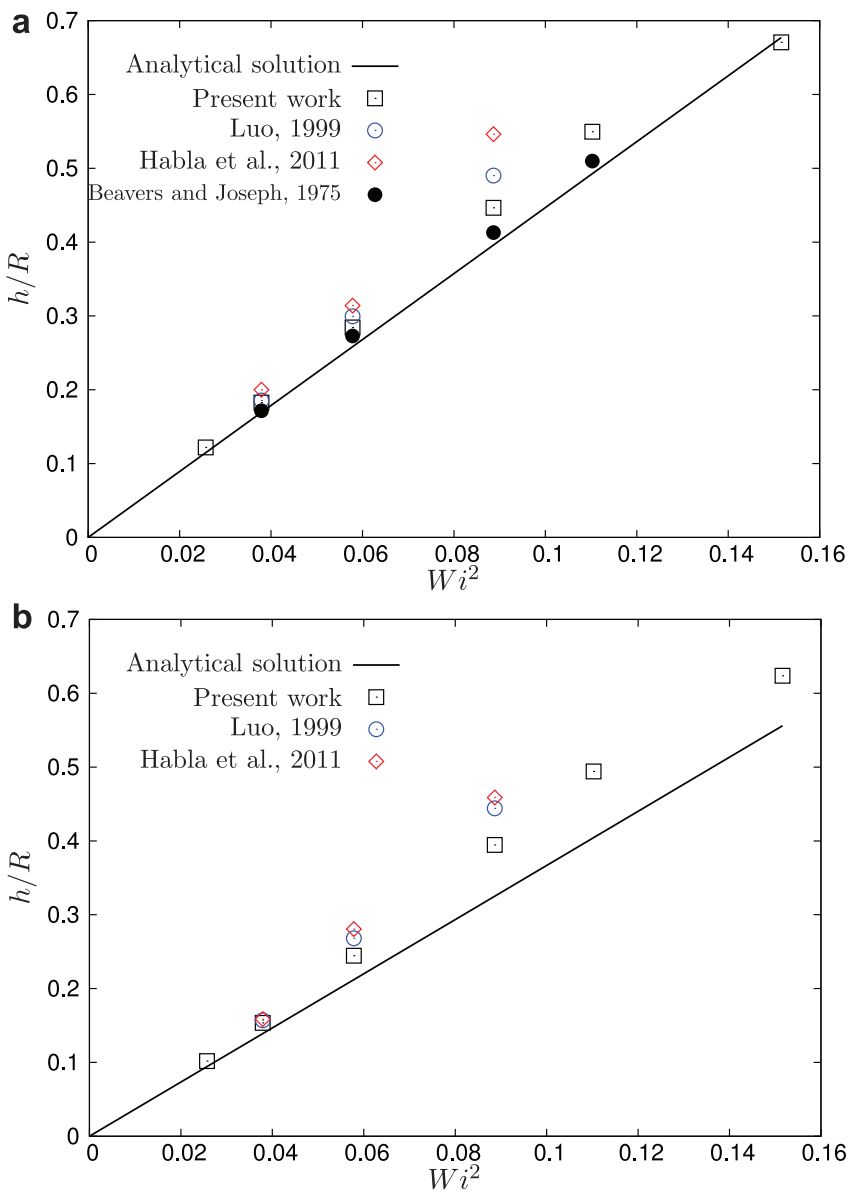

Fig. 11. Predicted climbing heights, $h$, compared with analytical (Beavers and Joseph, 1975) and numerical (Luo, 1999; Habla et al., 2011) results: (a) $\xi=0.284$ and (b) $\xi=0.318$.

$26^{\circ} \mathrm{C}, \hat{\beta}=1.02$, resulting in $\xi=0.318$ and $\xi=0.284$, respectively. The other phase was considered as a Newtonian fluid, with $\rho \rightarrow 0$ and $\mu_{s} \rightarrow 0$.

Fig. 11 presents the results for the maximum climbed height obtained for the proposed method, compared with the theoretical approximation (Eq. (30)) and experimental and numerical values proposed in the literature (Beavers and Joseph, 1975; Luo, 1999; Habla et al., 2011). We observe that both the numerical and experimental data differs from the perturbation theory for high angular velocities for both values of $\xi$. Nevertheless, one can see from Fig. 11 that our numerical results are closer to the experimental data (see Fig. 11a) and closer to the theoretical solution (see Fig. 11b).

Fig. 12 shows the numerical results obtained with the proposed method using the dimensionless parameters of Table 1. In addition, in these figures, we also plot the results presented by Beavers and Joseph (1975); Debbaut and Hocq (1992); Luo (1999); Habla et al. (2011). We can see that the increase of $\xi$ (from $\xi=0.284$ to $\xi=0.318$ ) leads to a decrease in the climbed height for all angular velocities. Similar results were obtained by Luo (1999); Habla et al. (2011). The difference in the climbing height for different $\xi$ values, is consistent with the theory whereby the Weissenberg effect also depends on the second normal stress differences. It can also be seen in Fig. 12 that as omega increases and consequently $W i$ increases, the climbing height increases. Furthermore, the comparison between the numerical results obtained with the proposed methodology and the results reported in the literature, showed good agreement. Fig. 12 also presents the results for the Oldroyd-B model $(\xi=0)$, which, for all angular velocities, presented higher climbing height, almost two times the climbing height obtained with the Johnson-Segalman model for higher $\xi, h_{\xi=0} / h_{\xi=0.318} \sim 2$.

The discrepancies between numerical and experimental data are more significant for higher angular velocities, as observed in Fig. $12 \mathrm{~d}$. This discrepancies can be rooted to the definition of the climbing constant $\hat{\beta}$. Since higher order terms are not considered in Eq. (30), it can feed some uncertainty on material parameters when using the Johnson-Segalman model to describe the fluid interface for large deformations. Notice that in Fig. 12d with $\xi=0.284$ to $\xi=0.318$, a bell shaped interface is formed. This is characterized as a consequence of a rapid slope interface change over a short distance, while for $\xi=0$, the interface is smoother, although presenting higher elevation.

\subsection{Flow dynamics at higher angular velocities}

The Weissenberg effect flow dynamics for high rod speeds have been studied experimentally by Joseph (1990); Beavers and Joseph (1979) and Degen et al. (1998). Beavers and Joseph (1979) observed different flow modes, depending on the rod velocities: for low $\omega$ the flow is steady; increasing $\omega$, other time-dependent modes were observed, including the breathing, nautilus, and rupture modes. Breathing is an axisymmetric rising and falling of the free surface of the climbing fluid parallel to the axis of the rod. The nautilus mode is an asymmetric structure with a spiral movement close to fluid surface. Finally, the rupture mode results in fluid being thrown around the container in a random fashion. Degen et al. (1998) presented an experimental work using the same fluid of Beavers and Joseph (1979), the STP oil additive, and defined three different regimes of the flow dynamics: fixed, periodic and aperiodic flows. The authors observed successive bifurcations from a time independent state to states with one frequency, two frequencies, three frequencies and then chaos, suggesting a characteristic Ruelle-Takens route to chaos.

As far as we are aware, such experimental flow features have not been reported in previous numerical studies. So, in order to obtain further insight into the fluid dynamics of the Weissenberg effect, and in particular its inherent unsteadiness, a new set of simulations was performed using the Oldroyd-B model and mesh M2. The full set of simulations and respective dimensionless groups are presented in Table 2.

As for the experimental works of Joseph (1990); Beavers and Joseph (1979) and Degen et al. (1998), the numerical simulations for the Oldroyd-B predicted steady free surface climbing for rod angular velocities lower than a critical velocity of $\omega_{c}=5.3 \mathrm{rev} / \mathrm{s}$. In Fig. 13, we can observe that increasing the rod rotation, the interfacial shape evolves from a smooth interface $(\omega=0.5 \mathrm{rev} / \mathrm{s})$ up to a bell shaped interface, with rapid curvature changes in the free surface over a short distance $(\omega=5.3 \mathrm{rev} / \mathrm{s})$. Further inspections in the simulations results allowed to correlate the interfacial shape evolution with the secondary motions within the climbing fluid. It is well known that in the Weissenberg effect flow experiment, inertia and the non-Newtonian extra stresses caused by the primary flow induce secondary motions. These secondary motions are depicted in Fig. 14 for $\omega=0.5 \mathrm{rev} / \mathrm{s}$ and $\omega=1.7 \mathrm{rev} / \mathrm{s}$. For low rotations, the secondary motion is composed of one large inertial vortex only with the fluid particles moving counterclockwise, while for high rotations a small secondary vortex appears near the rod, due to viscoelastic effects and normal stress differences, with the fluid particles moving clockwise, as already predicted theoretically by Yoo et al. (1979), numerically by Debbaut and Hocq (1992) and experimentally in Beavers and Joseph (1975) and Hu et al. (1990). We can also correlate the re-entrant point where the free surface changes curvature (see Fig. 14) with the line between the two opposed secondary motions. This point is of major importance, since 
a
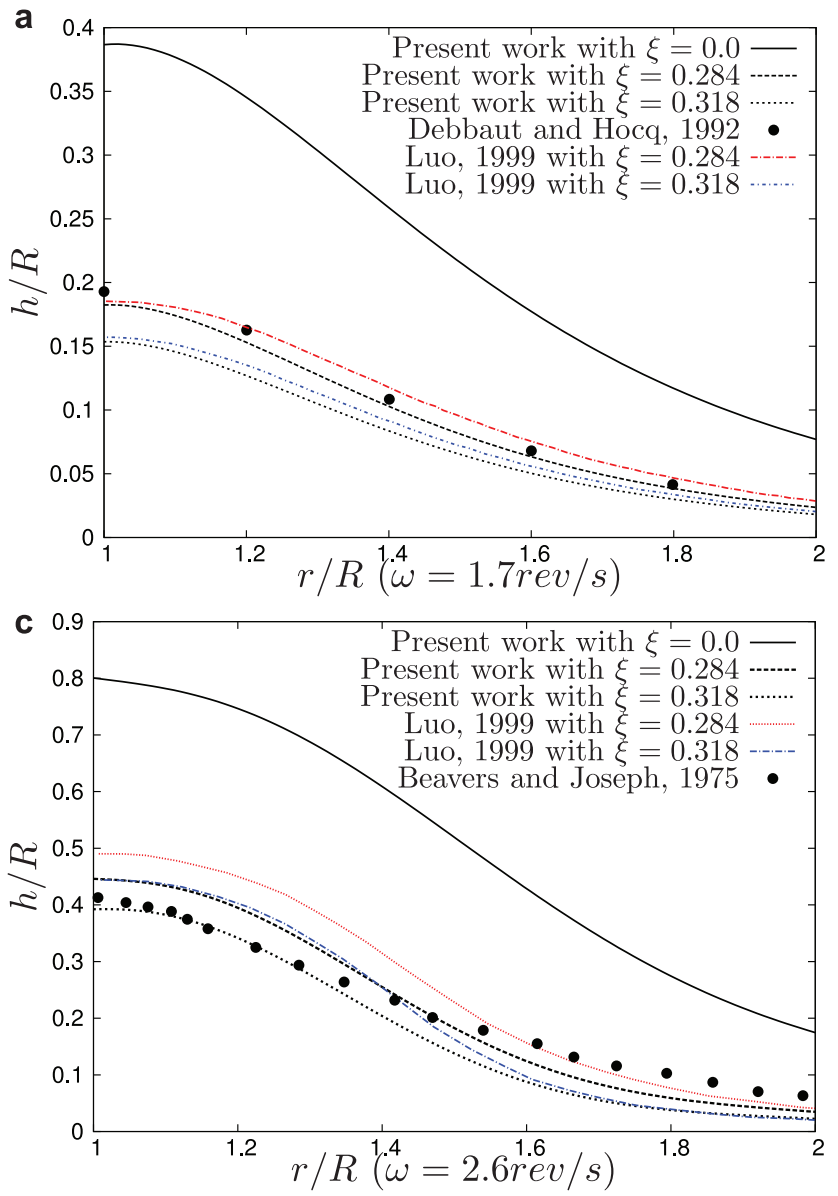

b
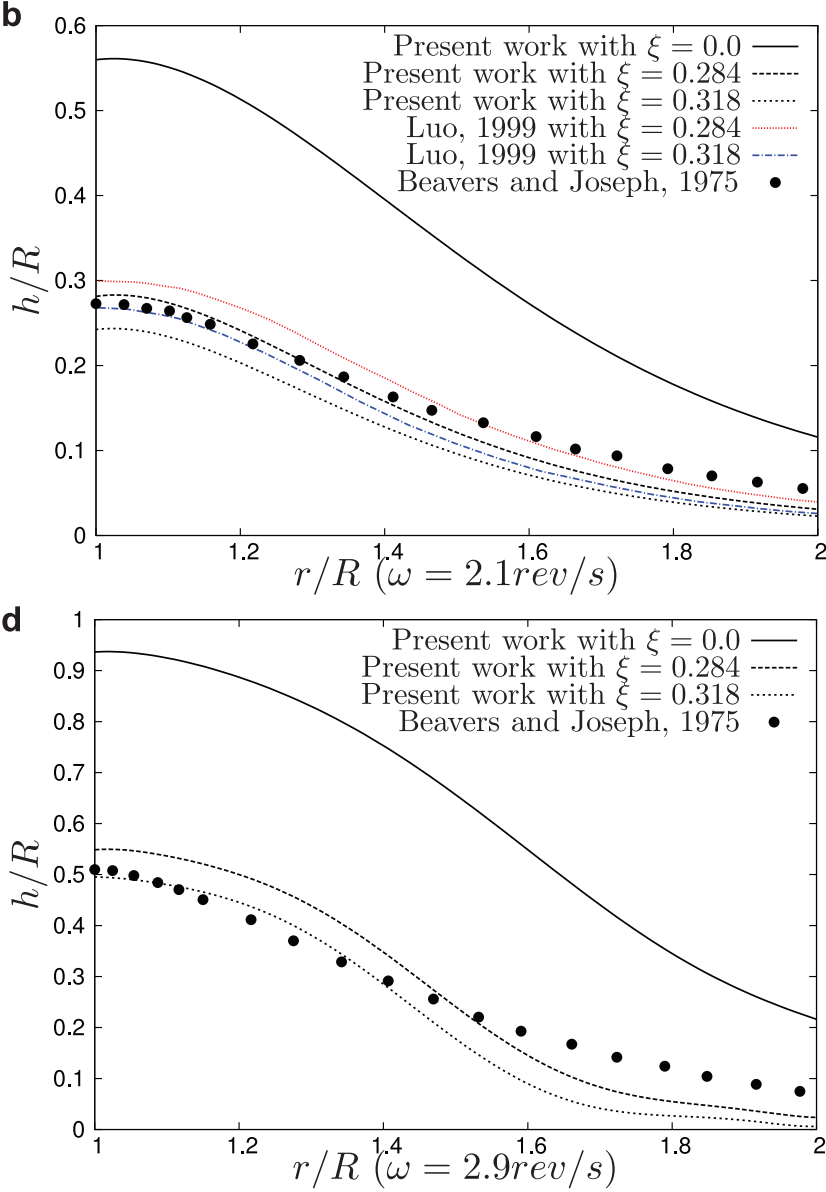

Fig. 12. Numerical results of the Weissenberg effect with $\xi=0.284$ and 0.318 : (a) $\omega=1.7 \mathrm{rev} / \mathrm{s}$, (b) $\omega=2.1 \mathrm{rev} / \mathrm{s}(\mathrm{c}) \omega=2.6 \mathrm{rev} / \mathrm{s}$ and $(\mathrm{d}) \omega=2.9 \mathrm{rev} / \mathrm{s}$.

Table 2

Angular velocity and dimensionless groups used to the study of the higher angular speeds.

\begin{tabular}{|c|c|c|c|c|}
\hline$\omega(\mathrm{rev} / \mathrm{s})$ & $W i$ & $R e$ & St & $\mathrm{Ca}$ \\
\hline 0.5 & 0.057 & 0.008 & 0.827 & 9.43 \\
\hline 1 & 0.115 & 0.015 & 1.655 & 18.85 \\
\hline 1.3 & 0.149 & 0.020 & 2.151 & 24.51 \\
\hline 1.5 & 0.172 & 0.023 & 2.482 & 28.28 \\
\hline 1.7 & 0.195 & 0.026 & 2.813 & 32.05 \\
\hline 2.1 & 0.240 & 0.032 & 3.475 & 39.59 \\
\hline 2.6 & 0.298 & 0.040 & 4.302 & 49.01 \\
\hline 2.9 & 0.332 & 0.045 & 4.798 & 54.67 \\
\hline 3.4 & 0.389 & 0.053 & 5.626 & 64.10 \\
\hline 4.0 & 0.458 & 0.062 & 6.619 & 75.41 \\
\hline 4.5 & 0.515 & 0.069 & 7.446 & 84.83 \\
\hline 5 & 0.573 & 0.077 & 8.273 & 94.26 \\
\hline 5.3 & 0.607 & 0.082 & 8.770 & 99.91 \\
\hline 5.5 & 0.630 & 0.085 & 9.100 & 103.68 \\
\hline 5.8 & 0.664 & 0.090 & 9.597 & 109.34 \\
\hline 6 & 0.687 & 0.093 & 9.928 & 113.11 \\
\hline 6.3 & 0.721 & 0.097 & 10.424 & 118.77 \\
\hline 6.5 & 0.744 & 0.100 & 10.755 & 122.54 \\
\hline 6.8 & 0.779 & 0.105 & 11.251 & 128.19 \\
\hline 7 & 0.802 & 0.108 & 11.582 & 131.96 \\
\hline 7.5 & 0.859 & 0.116 & 12.410 & 141.39 \\
\hline 7.8 & 0.893 & 0.121 & 12.906 & 147.04 \\
\hline 8 & 0.916 & 0.124 & 13.237 & 150.81 \\
\hline 8.3 & 0.950 & 0.128 & 13.733 & 156.47 \\
\hline 8.5 & 0.973 & 0.131 & 14.064 & 160.24 \\
\hline 8.6 & 0.985 & 0.134 & 14.230 & 162.12 \\
\hline 9 & 1.031 & 0.139 & 14.892 & 169.66 \\
\hline
\end{tabular}

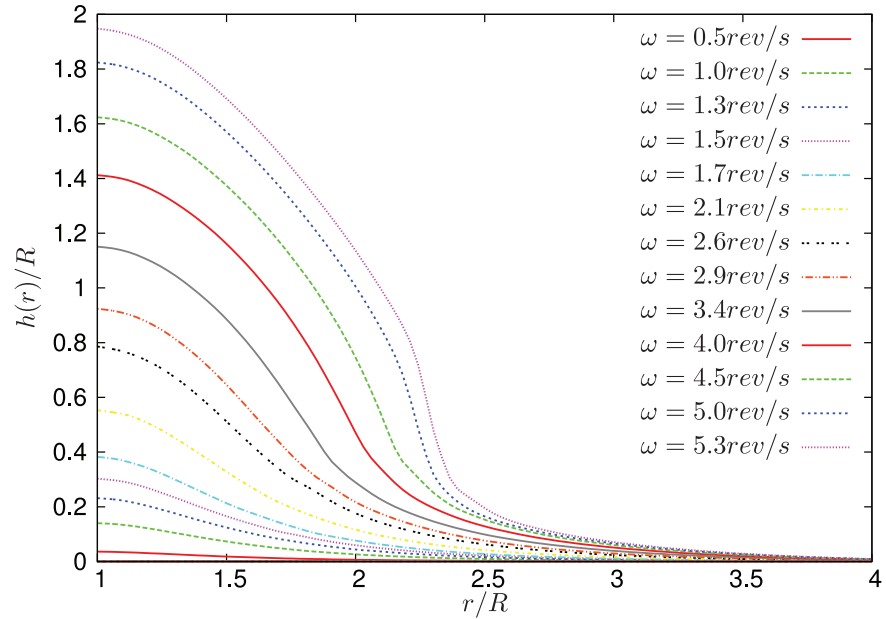

Fig. 13. Shape of the interface at different rod speeds, $0.5 \leq \omega \leq 5.3 \mathrm{rev} / \mathrm{s}$.

at high elasticity, rapid slope changes in the free surface can occur within an extremely short distance, and therefore, the interplay of elastic stresses, interfacial curvature and secondary flows can lead to the onset of tension-elastic instabilities.

Fig. 15 displays the dimensionless length (see the depicted sketch in Fig. 14b as a function of the rod angular velocity. From this picture, we can observe that the dimensionless length of the vortex increases monotonically for $\omega \geq 1.7 \mathrm{rev} / \mathrm{s}$ (Wi $\geq$ $0.195)$ while for $\omega \geq 4.0 \mathrm{rev} / \mathrm{s}(W i \geq 0.458)$ the dimensionless 


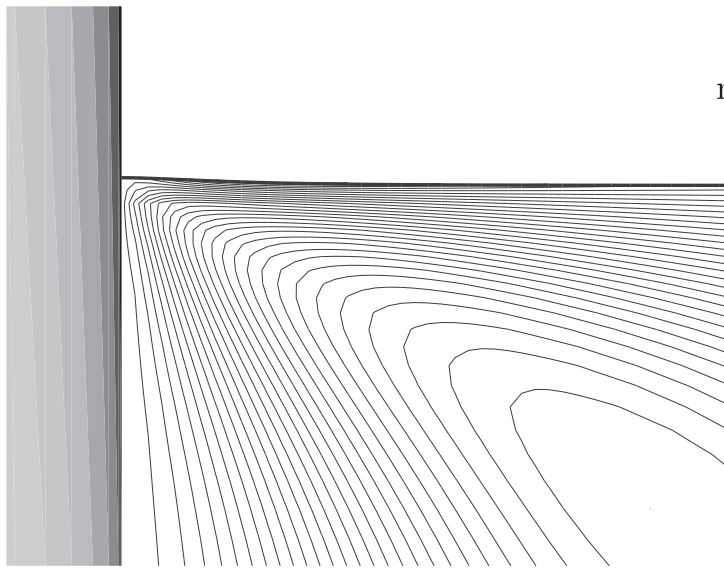

(a) $\omega=0.5 \mathrm{rev} / \mathrm{s}$

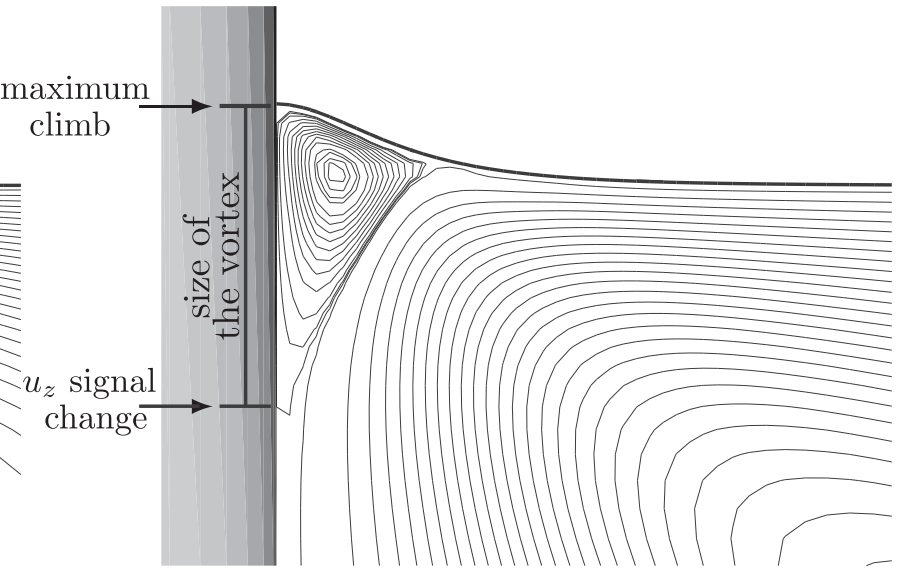

(b) $\omega=1.7 \mathrm{rev} / \mathrm{s}$

Fig. 14. Secondary flows and secondary vortex motions for the Weissenberg effect problem at $t=150$ s.

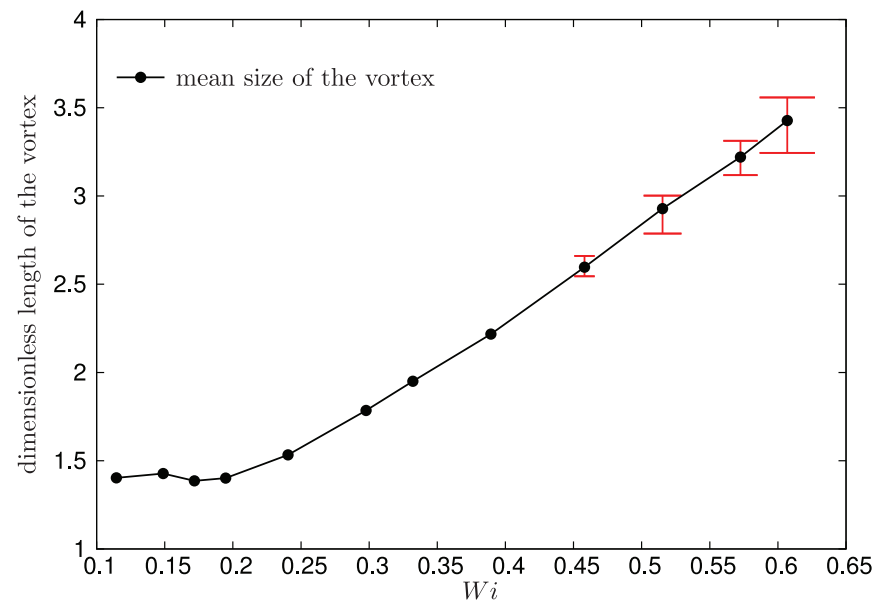

Fig. 15. Dimensionless length of the vortex for the range $1 \leq \omega \leq 5.3 \mathrm{rev} / \mathrm{s}$.

length of the vortex shows a time-periodic motion (the oscillations amplitude is marked as error bars in Fig. 15. The timeaveraged non-dimensional climbing height $h / R$ value as a function of the $W i$ is presented in Fig. 16a. Moreover, in this figure, we have illustrated the shape of free surface considering $\omega=$ $2.1 \mathrm{rev} / \mathrm{s}(W i=0.24), \omega=3.4 \mathrm{rev} / \mathrm{s}(W i=0.389), \omega=4 \mathrm{rev} / \mathrm{s}(W i=$ $0.458), \quad \omega=5 \mathrm{rev} / \mathrm{s}(W i=0.573), \quad \omega=5.8 \mathrm{rev} / \mathrm{s}(W i=0.664)$ and $\omega=9 \mathrm{rev} / \mathrm{s}(W i=1.031)$. According to Fig. 16a, the oscillations in the interface (measured by the variation of the climbing $h / R$ value and represented by error bars) start from $\omega>5.3 \mathrm{rev} / \mathrm{s}$ (Wi $>$ 0.607). Nevertheless, the vortex periodic motion did not affect the free surface shape up to $\omega>5.3 \mathrm{rev} / \mathrm{s}$.

As can we see in Fig. 16a, at larger values of the rotation speed, and above a critical value of $\omega_{c}=5.3 \mathrm{rev} / \mathrm{s}\left(W i_{c}=0.607\right)$ no steady free surface could be attained. Here, the plotted quantity for $\omega>5.3 \mathrm{rev} / \mathrm{s}$ is the time-averaged non-dimensional climbing value. Note that for slow rotations, the dependence of the climbing parameter $h / R$ upon $W i$ is quadratic, as predicted by the low order Eq. (30). The steady bell shaped interface configuration loses its stability to a time-periodic motion which initially appears with small amplitude and frequency, and increases in frequency as the rotational velocity is increased. This time-periodic motion is a bifurcating type of flow, whose amplitude increases with speed, as depicted in the amplitude graph of Fig. 16b. In this figure, the line represents the fitting to identify the critical $W i_{c}$ for an Hopf bifurcation to unsteady surface motion $\left(A=0.2492 \sqrt{W i-W i_{c}}\right.$ where
$A$ is the amplitude). This kind of bifurcation flow was also reported experimentally by Degen et al. (1998). Beavers and Joseph (1979) also presented experimental results for unsteady motions, called the "breathing instability".

We now report the most interesting results of this work, related to the numerical predictions for the breathing instability. Numerically, this time-periodic motion was well capture for values of $\omega \geq 5.3 \mathrm{rev} / \mathrm{s}$, as observed in the instantaneous plots of Figs. 17 and 18 , for $\omega=8 \mathrm{rev} / \mathrm{s}(W i=0.916)$ and $\omega=9 \mathrm{rev} / \mathrm{s}(W i=1.031)$, respectively. Improved understanding of the dynamics of the numerical breathing instability processes can be gained from the observation of supplementary movies available at supplementary material (see Electronic Annex 1 in the online version of this article). From Figs. 17 and 18 it can be observed that the portion of fluid enclosed in the second vortex rises slowly to a certain height, then collapses down into the body of fluid. The frequency and amplitude of this periodic motion increase as the rotational velocity is increased. In order to compare with the experimental results, a general description of the breathing instability can be found in Joseph and coworkers (Beavers and Joseph, 1975; Beavers et al., 1980) and in the photographs displayed in Fig. 95.3 by Joseph (1976) and in Fig. 17.13 by Joseph (1990). Experimentally, for the fluid used in their works at fluid bulk temperature of $25^{\circ} \mathrm{C}$, the critical timeperiodic motion was observed to be $\omega_{\operatorname{expc}}=4.94 \mathrm{rev} / \mathrm{s}$ and similar to the numerical value of $\omega_{c}=5.3 \mathrm{rev} / \mathrm{s}$. Simulation for higher rotation speeds were not performed, since experimentally the flow loses symmetry, presenting symmetry breaking instabilities, such as the nautilus and rupture modes. Full three-dimensional simulations will be performed in a future work in order to obtain realistic numerical predictions for these cases.

\section{Conclusions}

In this work a new viscoelastic multi-phase solver is presented. The solver is based on a finite difference scheme using the projection method to solve the governing equations of viscoelastic twophase flows. The interface between the fluids is approximated using the classical volume-of-fluid interface reconstruction algorithm, and a second-order operator-split method is used to solve the advection equation. Several viscoelastic models can be used to represent the rheological properties of the (multi)-fluid phases, employing appropriate matrix reformulations for the conformation tensor.

The solver numerical accuracy for distinct levels of physical complexities was assessed with several benchmark tests: the viscoelastic laminar lid-driven cavity flow, the 1D and 2D axisymmetric concentric annulus with inner cylinder rotation, the droplet 
a
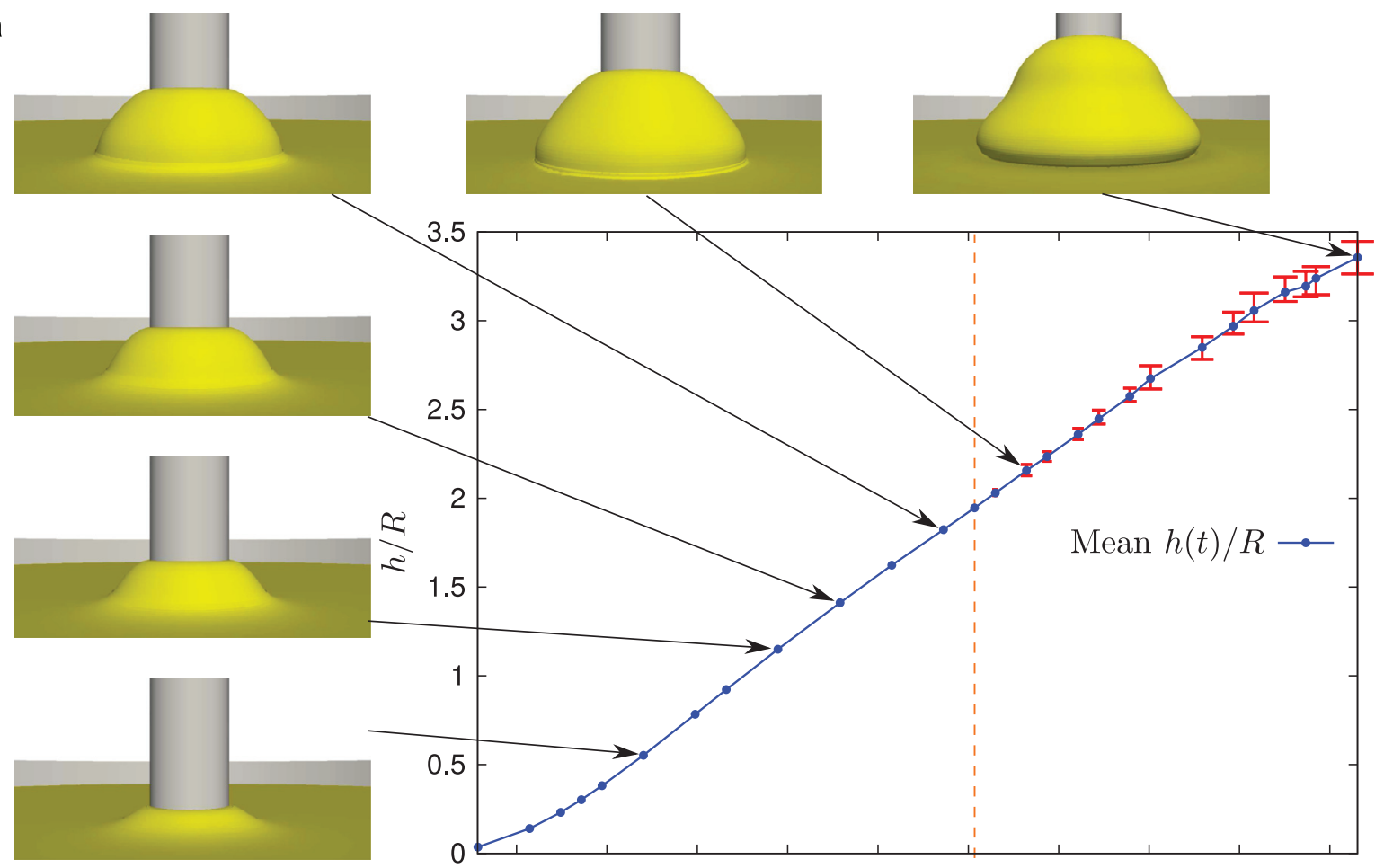

Mean $h(t) / R \rightarrow$

b

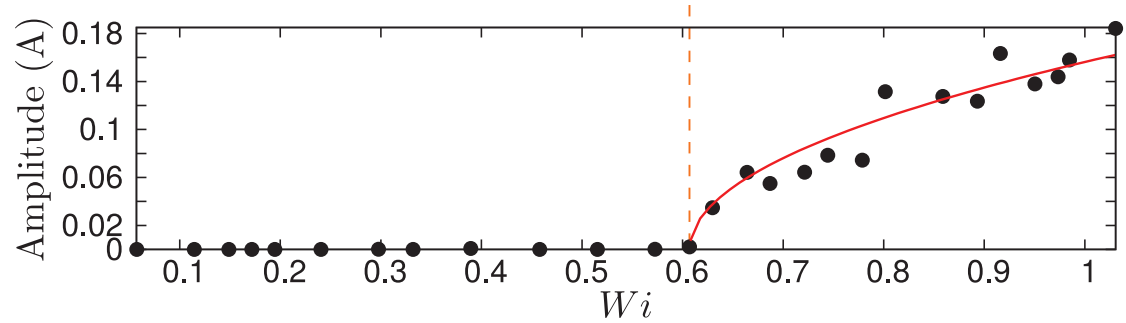

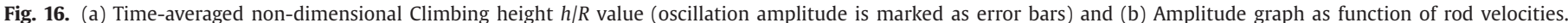
Line in the amplitude graph represents the fitting to identify the critical $W i_{c}$ for the Hopf bifurcation to breathing instability $\left(A=0.2492 \sqrt{W i-W i_{c}}\right)$.

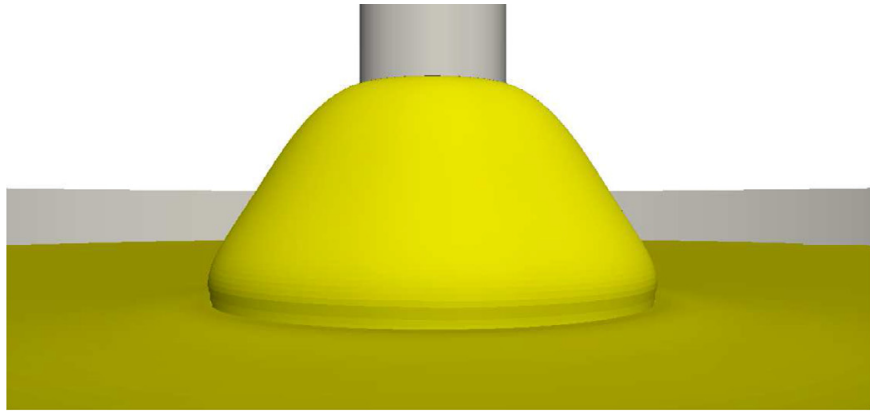

(a) $t \approx 142 \mathrm{~s}$

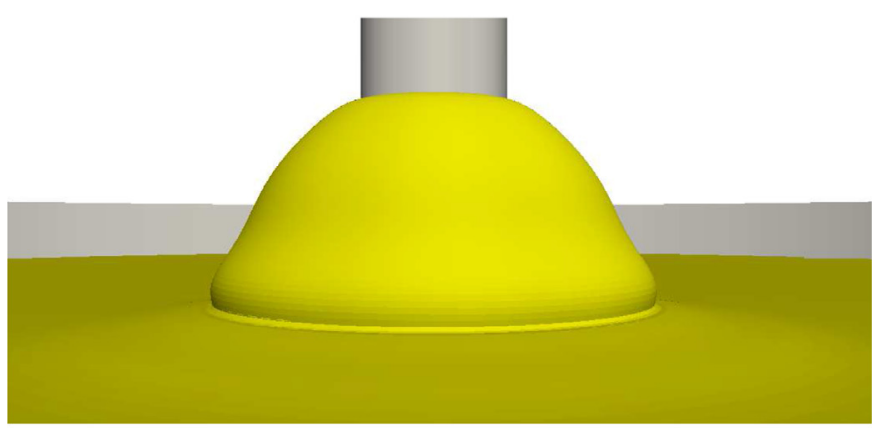

(c) $t \approx 147 \mathrm{~s}$

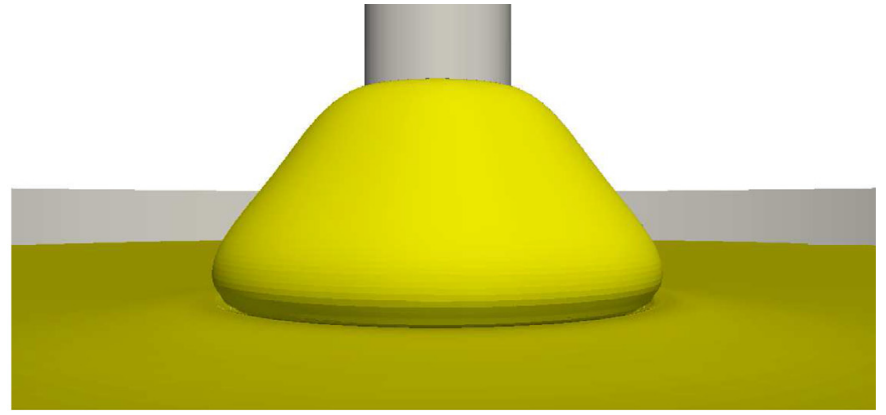

(b) $t \approx 145 \mathrm{~s}$

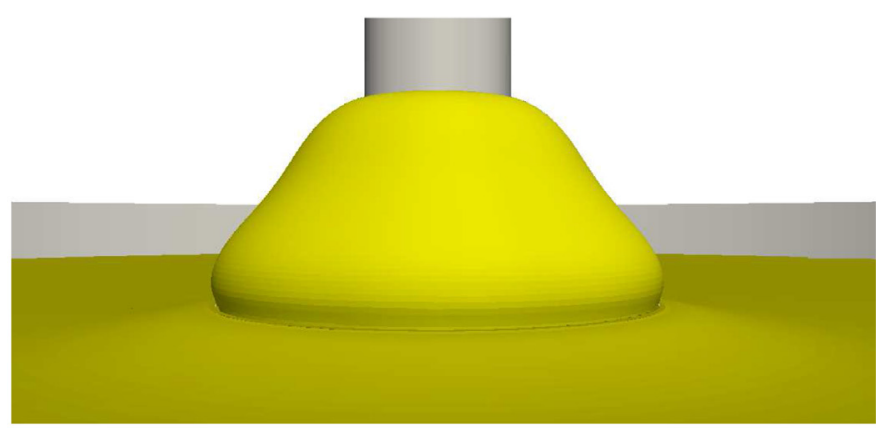

(d) $t \approx 149 \mathrm{~s}$

Fig. 17. Transient behavior of Weissenberg effect problem with $\omega=8 \mathrm{rev} / \mathrm{s}(W i=0.916)$. 


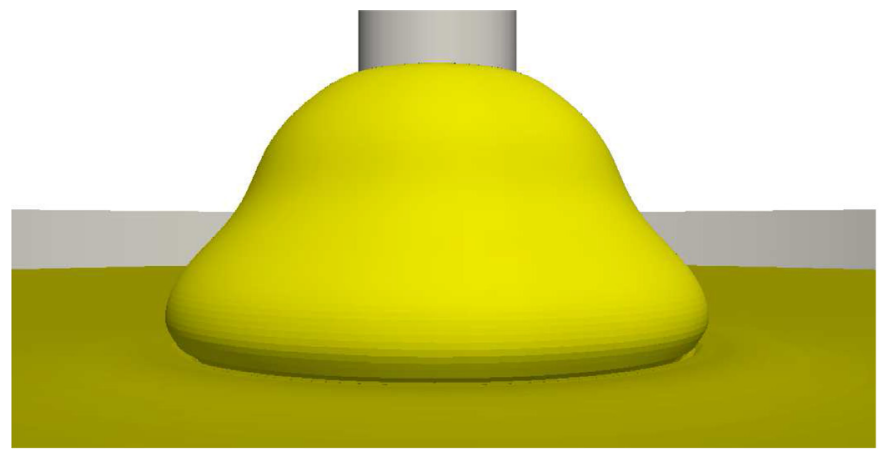

(a) $t \approx 185 \mathrm{~s}$

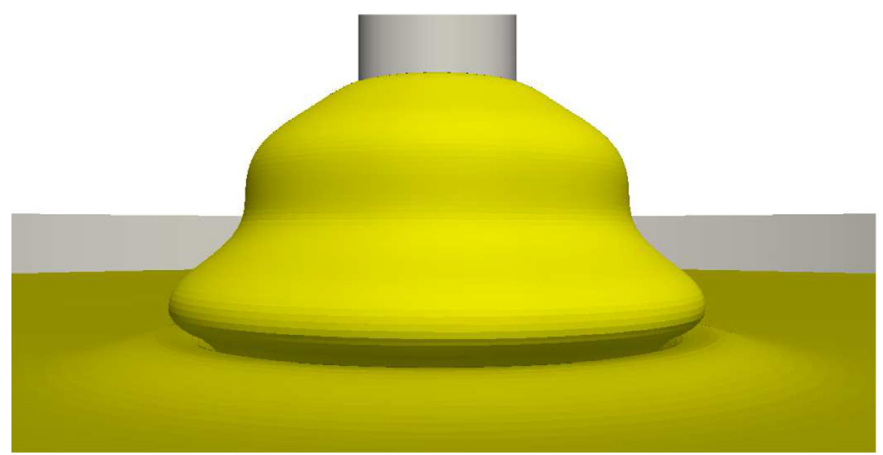

(c) $t \approx 195 \mathrm{~s}$

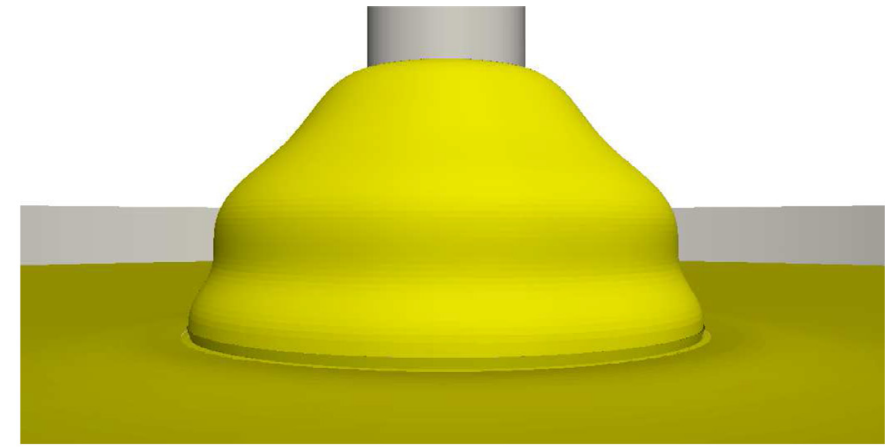

(b) $t \approx 190 \mathrm{~s}$

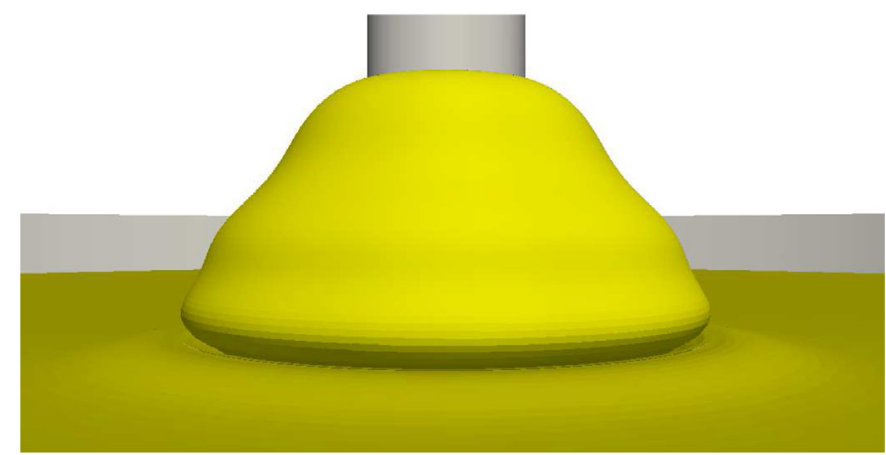

(d) $t \approx 200 \mathrm{~s}$

Fig. 18. Transient behavior of Weissenberg effect problem with $\omega=9 \mathrm{rev} / \mathrm{s}(W i=1.031)$.

formation and the droplet spreading of a viscoelastic fluid. For all the validation problems, the results showed an excellent agreement with literature data.

Finally, the Weissenberg effect was studied numerically, since it introduces all the effects validated previously. The results showed reasonable agreement with experimental and numerical results of the literature. Furthermore, above a critical value of the Weissenberg number, we were able to observe the onset of elastic instabilities driven by the combination of elastic stresses, interfacial curvature and secondary flows, that, as far as we are aware, such flow features have not been reported by previous numerical studies and it is the new enhanced capabilities of this new solver that made these predictions possible.

\section{Acknowledgments}

The authors acknowledge the financial support of the FAPESP (Fundação de Amparo à Pesquisa do Estado de São Paulo, grants 2013/07375-0, 2011/09194-7, 2015/04548-6 and CNPq (Conselho Nacional de Desenvolvimento Científico e Tecnológico, grant no. 309514/2013-4 and 473589/2013-3) which enabled this research to be carried out. A.M. Afonso would also like to thank Fundação para a Ciência e a Tecnologia (FCT), Portugal, for financial support through the scholarship SFRH/BPD/75436/2010.

\section{Appendix A. Interface representation and surface tension validation}

In this appendix, we present validations of the transport code and of the implementation of the surface tension.

\section{Transport of volume fraction: stretching of a circular fluid element}

To test the interface representation and volume fraction transport we rely on a benchmark test problem from the literature, namely the stretching of a circular fluid element. In this problem we use a circular fluid element placed in a swirling shear flow field within the $[0, \pi] \times[0, \pi]$ square. The circular fluid element with radius $0.2 \pi$ is centered at $(0.5 \pi, 0.2(\pi+1))$. The prescribed solenoidal velocity field is given by:

$$
\begin{aligned}
& \left.\begin{array}{l}
u(x, y, t)=\sin (x) \cos (y) \\
v(x, y, t)=-\cos (x) \sin (y)
\end{array}\right\} \text { for } \quad 0 \leq t \leq t_{f} / 2 \\
& \left.\begin{array}{l}
u(x, y, t)=-\sin (x) \cos (y) \\
v(x, y, t)=\cos (x) \sin (y)
\end{array}\right\} \text { for } \quad t_{f} / 2<t \leq t_{f},
\end{aligned}
$$

where $t_{f}$ represents the total simulation time. The velocity field (see Eq. (A.1) ) reverses the flow in half the time of the simulation, so that the final shape of the circular fluid element should be the initial shape considered in time $t=0 \mathrm{~s}$. This benchmark problem has been widely used in the literature, for instance Rudman (1997); Yokoi (2007).

Four uniform meshes were used for the computations: $M 1$ with $40 \times 40$ cells $(\Delta x=0.0785), M 2$ with $80 \times 80$ cells $(\Delta x=0.0393)$, M3 with $160 \times 160$ cells $(\Delta x=0.0196)$ and M4 with $320 \times 320$ cells $(\Delta x=0.00982)$. Fig. A.1 presents the results obtained with different meshes at three different simulation times: initial time, $t=0 \mathrm{~s}$, reversal time $t=t_{f} / 2=12 \mathrm{~s}$ and final time $t=t_{f}=24 \mathrm{~s}$. From this picture, we can observe that the final shape improves with mesh refinement. In addition, Table 3 presents the numerical volume, order of convergence and the error between the numerical and exact volume, calculated as

$E_{m}=\frac{V_{\text {exact }}-V_{\text {num }}}{V_{\text {exact }}} \times 100 \%$.

obtained at the end of a full cycle for all meshes. The volumetric error for the finest mesh is of order of $10^{-3} \%$, in agreement with other data from the literature (Rudman, 1997; Yokoi, 2007). 


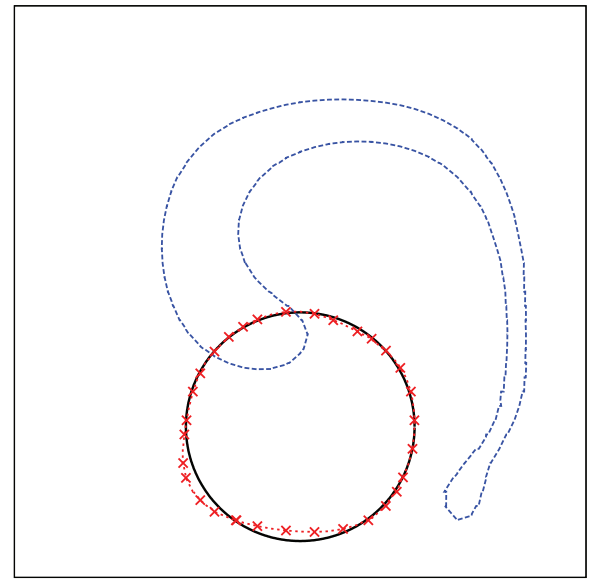

(a) Mesh $M 1$

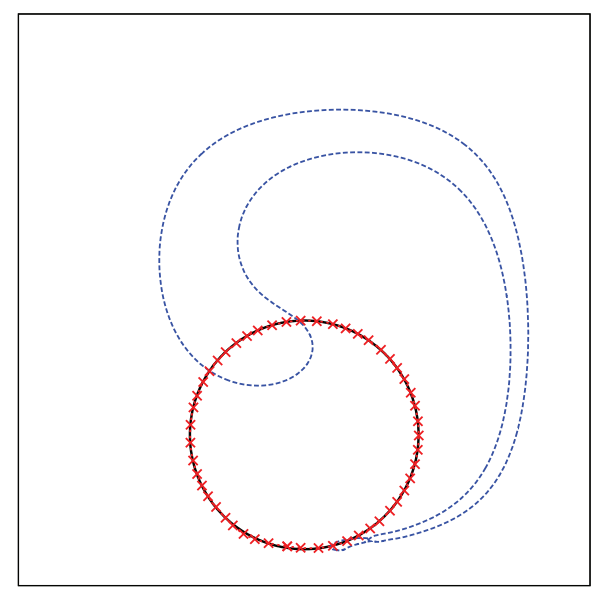

(c) Mesh $M 3$

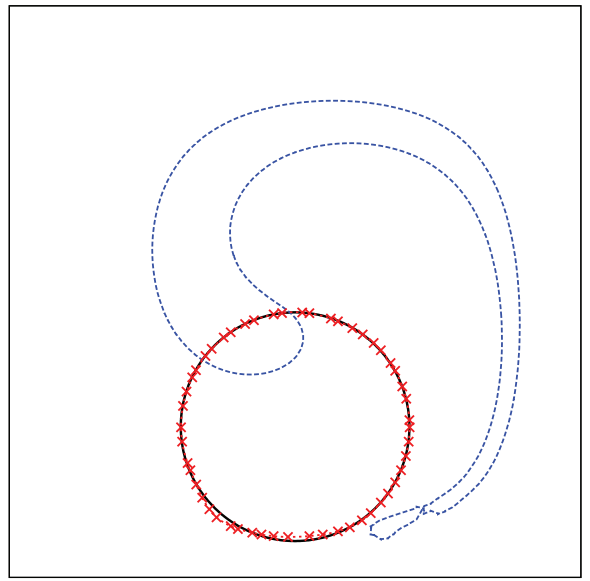

(b) Mesh $M 2$

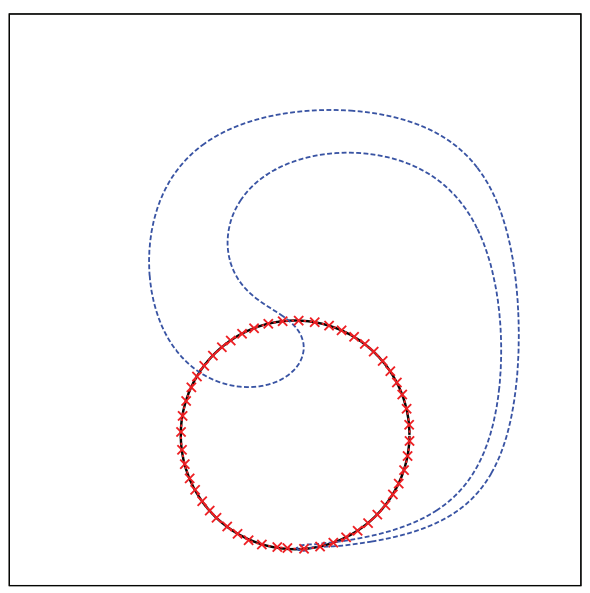

(d) Mesh M4

Fig. A.1. Deformation of a circulating fluid element at different times: solid line $-t=0 \mathrm{~s}$, dotted line $t=t_{f} / 2=12 \mathrm{~s}$ and line with crosses - end time $t=t_{f}=24 \mathrm{~s}$.

Table 3

Simulation results of the deformation of a circular fluid element: Numeric volume $V_{n u m}$, volumetric error $E_{m}$ and order of convergence.

\begin{tabular}{llll}
\hline Mesh & $V_{\text {num }}$ & $E_{m} \%$ & Order \\
\hline M1 & 1.23742 & $2.83189 \times 10^{-1}$ & - \\
M2 & 1.23946 & $7.91949 \times 10^{-2}$ & 1.84 \\
M3 & 1.24004 & $2.11092 \times 10^{-2}$ & 1.90 \\
M4 & 1.24020 & $5.61272 \times 10^{-3}$ & 1.91 \\
\hline
\end{tabular}

\section{Surface tension computations: Newtonian oscillating droplet}

In this second benchmark problem we examine the accuracy of the surface tension body force implementation. In this test, the surface tension body force, $\mathbf{F}$ in Eq. (2), is activated. This term is implemented using the CSF approximation method, while the curvature, defined by Eq. (15), is approximated according to the Height Function method.

In this benchmark test, also studied by Montefuscolo et al. (2014) using ALE methods and by Watanabe (2008) using a conservative Level-Set method, we consider an initial disturbed spherical Newtonian droplet and measure the instantaneous shape deformation along time. The droplet is embedded in a Newtonian fluid phase with $\rho \rightarrow 0$ and $\mu \rightarrow 0$. Here, we assume that the shape oscillations are axisymmetric, and adopt a spherical coordinate system $(r ; \psi ; \theta)$. The initial drop deformation, $r(\theta)$, can be expressed in terms of the Legendre polynomial, $P_{n}(\cos \theta)$, of order 2 and amplitude $10 \%$ of its radius $(\epsilon=0.1)$ :

$r(\theta)=R_{0}\left[1+\epsilon P_{n}(\cos \theta)\right]$

where $R_{0}$ is the radius of the unperturbed drop. The generalized linear solution of the problem, including the influence of a surrounding fluid, was presented by Lamb (1932). This solution describes the instantaneous deformation of the droplet shape by an infinite series of the surface spherical harmonics, where each term of this function corresponds to one independent natural oscillation mode.

To assess the mesh convergence, the simulations for the oscillating droplet were performed in three uniform meshes: $M 1$ with $64 \times 64$ cells $(\Delta r=\Delta z=1 / 64), M 2$ with $128 \times 128$ cells $(\Delta r=$ $\Delta z=1 / 128)$ and $M 3$ with $256 \times 256$ cells $(\Delta r=\Delta z=1 / 256)$. The time step size is determined so as to satisfy the CFL conditions due to surface tension and viscous terms. The Reynolds number, $R e=\rho \sigma R / \mu^{2}$, and the Ohnesorge number, $O h=\mu / \sqrt{\rho R \sigma}$, are fixed at 13.15 and 0.0195 , respectively. For low values of the Ohnesorge number, the drop exhibits weakly-damped oscillations, with a period and decay time that can be estimated as $\rho \pi^{2} R^{3} /(2 \sigma)$ and $\rho R^{2} /(5 \mu)$, respectively, and the instantaneous deformation amplitude can be approximated by a pseudo-analytical solution 


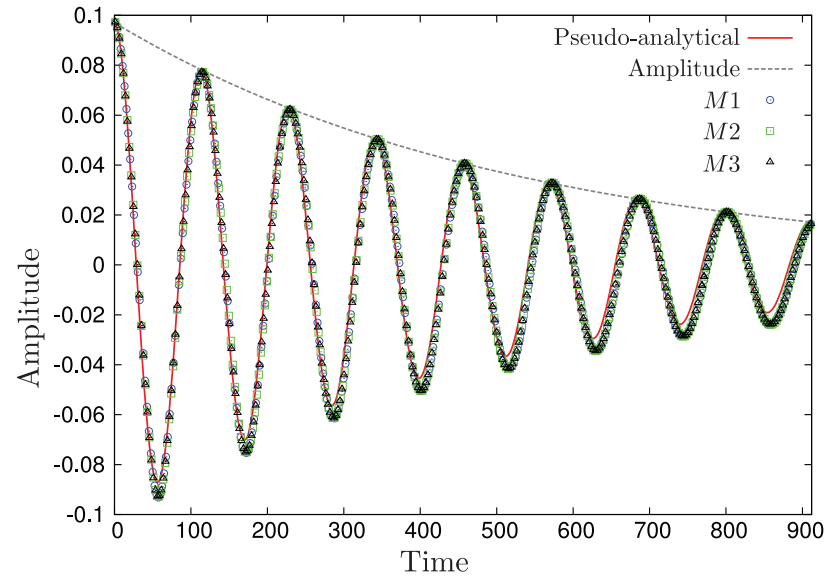

Fig. A.2. Numerical and theoretical time evolution (and Lamb's (Lamb, 1932) linear theory for decay of amplitude) of a natural droplet oscillation.

(Montefuscolo et al., 2014), which will be used here for benchmarking. For this test, at $r=0$ and $z=0$ we have adopted the symmetry boundary conditions while on the right and top boundaries homogeneous Neumann boundary conditions are imposed for all fields.

The numerical and theoretical time evolution for amplitude is presented in Fig. A.2, along with the results obtained using Lamb's linear theory Lamb (1932). We can observe that for all meshes, the agreement between the numerical and the theoretical results is quite good, with a perfect match with the expected Lamb's linear theory. The numerical and theoretical oscillation period is $T=114.68$ and $T=113.92$, respectively. Given that the pseudoanalytical solution (Montefuscolo et al., 2014) is only valid for infinitesimal amplitudes, therefore some differences are observed, reflected in the small shift in the oscillation frequencies for longer times.

\section{Supplementary material}

Supplementary material associated with this article can be found, in the online version, at 10.1016/j.ijmultiphaseflow.2016.04. 014

\section{References}

Afonso, A.M., Pinho, F.T., Alves, M.A., 2012. The kernel-conformation constitutive laws. J. Non-Newton. Fluid. 167-168, 30-37. doi:10.1016/j.jnnfm.2011.09.008.

Beavers, G.S., Joseph, D.D., 1975. The rotating rod viscometer. J. Fluid Mech. 69, 475511. doi: $10.1017 /$ S002211207500153X.

Beavers, G.S., Joseph, D.D., 1979. Experiments on free surface phenomena. J. NonNewton. Fluid. 5, 323-352. doi:10.1016/0377-0257(79)85022-3.

Beavers, G.S., Yoo, J.Y., Joseph, D.D., 1980. The free surface on a liquid between cylinders rotating at different speeds. part III. Rheol. Acta 19, 19-31. doi:10.1007/ BF01523851.

Bird, R.B., Armstrong, R.C., Hassager, O., 1987. Dynamics of polymeric liquids. Fluid mechanics, 1. John Wiley and Sons Inc.

Brackbill, J.U., Kothe, D.B., Zemach, C., 1992. A continuum method for modeling surface tension. J. Comput. Phys. 100, 335-354. doi:10.1016/0021-9991(92)90240-Y.

Chinyoka, T., Renardy, Y.Y., Renardy, M., Khismatullin, D.B., 2005. Two-dimensional study of drop deformation under simple shear for O ldroyd-b liquids. J. NonNewton. Fluid. 130, 45-56. doi:10.1016/j.jnnfm.2005.07.005.

Debbaut, B., Hocq, B., 1992. On the numerical simulation of axisymmetric swirling flows of differential viscoelastic liquids: the rod climbing effect and the quelleffekt. J. Non-Newton. Fluid. 43, 103-126. doi:10.1016/0377-0257(92)80019-T.

Degen, M.M., Kahle, W.J., Andereck, C.D., 1998. Time-dependent states in the weissenberg effect. Phys. Rev. E 57.2, 1761. doi:10.1103/PhysRevE.57.1761.

Denner, F., van Wachem, B.G.M., 2015. Numerical time-step restrictions as a result of capillary waves. J. Comput. Phys. 285, 24-40. doi:10.1016/j.jcp.2015.01.021.

Escudier, M.P., Oliveira, P.J., Pinho, F.T., 2002. Fully developed laminar flow of purely viscous non-newtonian liquids through annuli including the effects of eccentricity and inner-cylinder rotation. Int. J. Heat Fluid Fl. 23, 52-73. doi:10.1016 S0142-727X(01)00135-7.
Fattal, R., Kupferman, R., 2005. Time-dependent simulation of viscoelastic flows at high weissenberg number using the log-conformation representation. J. NonNewton. Fluid. 126, 23-37. doi:10.1016/j.jnnfm.2004.12.003.

Ferziger, J.H., Peric, M., 2012. Computational Methods for Fluid Dynamics.. Springer Science \& Business Media.

Figueiredo, R.A., Oishi, C.M., Cuminato, J.A., Alves, M.A., 2013. Three-dimensional transient complex free surface flows: Numerical simulation of XPP fluid. J. NonNewton. Fluid. 195, 88-98. doi:10.1016/j.jnnfm.2013.01.004.

Figueiredo, R.A., Oishi, C.M., Cuminato, J.A., Azevedo, J.C., Afonso, A.M., Alves, M.A., 2014. Numerical investigation of three dimensional viscoelastic free surface flows: impacting drop problem. In: Proceeding of 6th European Conference on Computational Fluid Dynamics.

Francois, M.M., Cummins, S.J., Dendy, E.D., Kothe, D.B., Sicilian, J.M., Williams, M.W. 2006. A balanced-force algorithm for continuous and sharp interfacial surface tension models within a volume tracking framework. J. Comput. Phys. 213, 141173. doi:10.1016/j.jcp.2005.08.004.

Giesekus, H., 1982. A simple constitutive equation for polymer fluids based on the concept of deformation-dependent tensional mobility. J. Non-Newton. Fluid. 11, 69-109. doi:10.1016/0377-0257(82)85016-7.

Habla, F., Marschall, H., Hinrichsen, O., Dietsche, L., Jasak, H., Favero, J.L., 2011. Numerical simulation of viscoelastic two-phase flows using openFOAM. Chem. Eng. Sci. 66, 5487-5496. doi:10.1016/j.ces.2011.06.076.

Habla, F., Tan, M.W., Hablberger, J., Hinrichsen, O., 2014. Numerical simulation of the viscoelastic flow in a three-dimensional lid-driven cavity using the logconformation reformulation in openFOAM. J. Non-Newton. Fluid. 212, 47-62. doi:10.1016/j.jnnfm.2014.08.005.

Hirt, W., Nichols, B.D., 1981. Volume of fluid (VOF) methods for the dynamics of free boundaries. J. Comput. Phys. 39, 201-225. doi:10.1016/0021-9991(81)90145-5.

Hoang, D.A., van Steijn, V., Portela, L.M., Kreutzer, M.T., Kleijn, C.R., 2013. Benchmark numerical simulations of segmented two-phase flows in microchannels using the volume of fluid method. Comput. Fluids 86, 28-36. doi:10.1016/j.compfluid. 2013.06.024.

Hu, H.H., Riccius, O., Chen, K.P., Arney, M., Joseph, D.D., 1990. Climbing constant, second-order correction of troutons viscosity, wave speed and delayed die swell for M1. J. Non Newton. Fluid Mech. 35, 287-307.

Izbassarov, D., Muradoglu, M., 2015. A front-tracking method for computational modeling of viscoelastic two-phase flow system. J. Non Newton. Fluid. 223, 122 140. doi:10.1016/j.jnnfm.2015.05.012.

Johnson Jr., M.W., Segalman, D., 1977. A model for viscoelastic fluid behavior which allows non-affine deformation. J. Non Newton. Fluid. 2, 255-270. doi:10.1016/ 0377-0257(77)80003-7.

Joseph, D.D., 1976. Stability of Fluid Motions II.. Springer Tracts in Natural Philosophy, 28. Springer-Verlag, Berlin.

Joseph, D.D., 1990. Fluid Dynamics of Viscoelastic Liquids.. Applied Mathematical Science Series, 84. Springer-Verlag, Berlin, pp. 510-514.

Joseph, D.D., Beavers, G.S., Cers, A., Dewald, C., Hoger, A., Phan, P.T., 1984. Climbing constants for various liquids. J. Rheol. 28, 325-345. doi:10.1122/1.549754

Khismatullin, D., Renard, Y., Renardy, M., 2006. Development and implementation of VOF-PROST for 3d viscoelastic liquid-liquid simulations. J. Non Newton. Fluid. 140, 120-131. doi:10.1016/j.jnnfm.2006.02.013.

Lamb, H., 1932. Hydrodynamics. Cambridge University Press.

Lind, S.J., Phillips, T.N., 2010. The effect of viscoelasticity on a rising gas bubble. J. Non-Newton. Fluid. 165, 852-865. doi:10.1016/j.jnnfm.2010.04.002.

Luo, X.L., 1999. Numerical simulation of Weissenberg phenomena - the rodclimbing of viscoelastic fluids. Comput. Method Appl. M. 180, 393-412. doi:10. 1016/S0045-7825(99)00175-9.

Martins, F.P., Oishi, C.M., Afonso, A.M., Alves, M.A., 2015. A numerical study of the Kernel-conformation transformation for transient viscoelastic fluid flows. J. Comput. Phys. 302, 653-673. doi:10.1016/j.jcp.2015.08.038.

Mirzazadeh, M., Escudier, M.P., Rashidi, F., Hashemabadi, S.H., 2005. Purely tangential flow of a PTT-viscoelastic fluid within a concentric annulus. J. Non-Newton. Fluid. 129, 88-97. doi:10.1016/j.jnnfm.2005.05.009.

Montefuscolo, F., Sousa, F.S., Buscaglia, G.C., 2014. High-order ALE schemes for incompressible capillary flows. J. Comput. Phys. 278, 133-147. doi:10.1016/j.jcp. 2014.08.030.

Osher, S., Fedkiw, R., 2001. Level set methods: An overview and some recent results. J. Comput. Phys. 169, 463-502. doi:10.1006/jcph.2000.6636.

Palhares-Junior, I.L., Oishi, C.M., Afonso, A.M., Alves, M.A., Pinho, F.T., 2016. Numerical study of the square-root conformation tensor formulation for confined and free-surface viscoelastic fluid flows. Adv. Model. Simul. Eng. Sci. 3, 1-23. doi:10.1186/s40323-015-0054-4.

Pan, T.-W., Hao, J., Glowinski, R., 2009. On the simulation of a time-dependent cavity flow of an Oldroyd-b fluid. Int. J. Numer. Meth. Fl. 60, 791-808. doi:10.1002/fld. 1919.

Phan-Thien, N., Tanner, R.I., 1977. A new constitutive equation derived from network theory. J. Non Newton. Fluid. 2, 353-365. doi:10.1016/0377-0257(77)80021-9.

Van der Pijl, S.P., Segal, A., Vuik, C., Wesseling, P., 2005. A mass-conserving level-set method for modelling of multi-phase flows. Int. J. Numer. Meth. Fl. 47, 339-361. doi:10.1002/fld.817.

Pillapakkam, S.B., Singh, P., 2001. A level-set method for computing solutions to viscoelastic two-phase flow. J. Comput. Phys. 174, 552-578. doi:10.1006/jcph.2001. 6927.

Pilliod, J.E., Puckett, E.G., 2004. Second-order accurate volume-of-fluid algorithms for tracking material interfaces. J. Comput. Phys. 199, 465-502. doi:10.1016/j. jcp.2003.12.023. 
Pinho, F.T., Oliveira, P.J., 2000. Axial annular flow of a non-linear viscoelastic fluid-an analytical solution. J. Non Newton. Fluid. 93, 325-337. doi:10.1016/ S0377-0257(00)00113-0.

Pivello, M.R., Villar, M.M., Serfaty, R., Roma, A.M., Silveira-Neto, A., 2014. A fully adaptive front tracking method for the simulation of two phase flows. Int. J. Multiphas. Flow 58, 72-82. doi:10.1016/j.ijmultiphaseflow.2013.08.009.

Quan, S., 2011. Simulations of multiphase flows with multiple length scales using moving mesh interface tracking with adaptive meshing. J. Comput. Phys. 230, 5430-5448. doi:10.1016/j.jcp.2011.03.050.

Raessi, M., Bussmann, M., Mostaghimi, J., 2009. A semi-implicit finite volume implementation of the CSF method for treating surface tension in interfacial flows. Int. J. Numer. Meth. Fl. 59, 1093-1110. doi:10.1002/fld.1857.

Ravanchi, M.T., Mirzazadeh, M., Rashidi, F., 2007. Flow of Giesekus viscoelastic fluid in a concentric annulus with inner cylinder rotation. Int. J. Heat Fluid Fl. 28-4, 838-845. doi:10.1016/j.ijheatfluidflow.2006.08.003.

Rudman, M., 1997. Volume-tracking methods for interfacial flow calculations.. Int. J. Numer. Meth. Fl. 24, 671-691.

Scardovelli, R., Zaleski, S., 1999. Direct numerical simulation of free-surface and interfacial flow. Annu. Rev. Fluid Mech. 31, 567-603. doi:10.1146/annurev.fluid.31. 1.567.

Stewart, P.A., Lay, N., Sussman, M., Ohta, M., 2008. An improved sharp interface method for viscoelastic and viscous two-phase flows. J. Sci. Comput. 35, 43-61. doi:10.1007/s10915-007-9173-5.

Su, J., Ouyang, J., Wang, X., Yang, B., Zhou, W., 2013. Lattice Boltzmann method for the simulation of viscoelastic fluid flows over a large range of Weissenberg numbers. J. Non-Newton. Fluid. 194, 42-59. doi:10.1016/j.jnnfm.2012.11.006.

Sussman, M., Fatemi, E., Smereka, P., Osher, S., 1998. An improved level set method for incompressible two-phase flows. Comput. Fluids 27, 663-680. doi:10.1016 S0045-7930(97)00053-4.
Sussman, M., Puckett, E.G., 2000. A coupled level set and volume-of-fluid method for computing 3d and axisymmetric incompressible two-phase flows. J. Comput. Phys. 162, 301-337. doi:10.1006/jcph.2000.6537.

Sussman, M., Smereka, P., Osher, S., 1994. A level set approach for computing solutions to incompressible two-phase flow. J. Comput. Phys. 114, 146-159. doi:10.1006/jcph.1994.1155.

Sussman, M., Smith, K., Hussaini, M., Ohta, M., Zhiwei, R., 2007. A sharp interface method for incompressible two-phase flows. J. Comput. Phys. 221, 469-505. doi:10.1016/j.jcp.2006.06.020.

Tryggvason, G., Bunner, B., Esmaeeli, A., Juric, D., Al-Rawahi, N., Tauber, W., Han, J., Nas, S., Jan, Y.-J., 2001. A front-tracking method for the computations of multiphase flow. J. Comput. Phys. 169, 708-759. doi:10.1006/jcph.2001.6726.

Watanabe, T., 2008. Numerical simulation of oscillations and rotations of a free liquid droplet using the level set method. Comput. Fluids 37, 91-98. doi:10.1016/j. compfluid.2007.04.004

Xu, X., Ouyang, J., Jiang, T., Li, Q., 2012. Numerical simulation of 3d-unsteady viscoelastic free surface flows by improved smoothed particle hydrodynamics method. J. Non Newton. Fluid. 177-178, 109-120. doi:10.1016/j.jnnfm.2012.04. 006

Yokoi, K., 2007. Efficient implementation of THINC scheme: A simple and practical smoothed VOF algorithm. J. Comput. Phys. 226, 1985-2002. doi:10.1016/j. jcp.2007.06.020.

Yoo, Y., Joseph, D.D., Beavers, G.S., 1979. Higher order theory of the weissenberg effect.. J. Fluid Mech. 92, 529-590. doi:10.1017/S0022112079000768.

Youngs, D.L., 1982. Time-Dependent Multi-Material Flow with Large Fluid Distortion. Numerical Methods for Fluid Dynamics. Academic Press, pp. 273-285.

Yue, P., Feng, J.J., Liu, C., Shen, J., 2005. Viscoelastic effects on drop deformation in steady shear. J. Fluid Mech. 540, 427-437. doi:10.1017/S0022112005006166. 